\title{
Channel Equalization for Single Carrier MIMO Underwater Acoustic Communications
}

\author{
Jun Tao, ${ }^{1}$ Yahong Rosa Zheng, ${ }^{2}$ Chengshan Xiao, ${ }^{2}$ T. C. Yang, ${ }^{3}$ and Wen-Bin Yang ${ }^{4}$ \\ ${ }^{1}$ Department of Electrical \& Computer Engineering, University of Missouri, Columbia, MO 65211, USA \\ ${ }^{2}$ Department of Electrical \& Computer Engineering, Missouri University of Science \& Technology, Rolla, MO 65409, USA \\ ${ }^{3}$ Acoustic Division, Naval Research Laboratory, Washington, DC 20375, USA \\ ${ }^{4}$ Advanced Network Technologies Division, Information Technology Laboratory, National Institute of Standards and Technology, \\ Gaithersburg, MD 20899, USA
}

Correspondence should be addressed to Chengshan Xiao, xiaoc@mst.edu

Received 28 October 2009; Revised 8 April 2010; Accepted 18 May 2010

Academic Editor: Ananthram Swami

Copyright (C) 2010 Jun Tao et al. This is an open access article distributed under the Creative Commons Attribution License, which permits unrestricted use, distribution, and reproduction in any medium, provided the original work is properly cited.

\begin{abstract}
Multiple-input multiple-output (MIMO) underwater acoustic (UWA) channels introduce both space-time interference (STI) and time-varying phase distortion for transmitted signals. In such cases, the equalized symbols produced by conventional equalizer aiming for STI cancelation suffer phase rotation and thus cannot be reliably detected. In this paper, we propose a new equalization scheme for high data rate single carrier MIMO UWA channels. Different from existing methods employing joint equalization and symbolwise phase tracking technology, the proposed scheme decouples the interference cancelation (IC) operation and the phase compensation operation, leading to a generalized equalizer structure combining an IC equalizer with a phase compensator. The decoupling of the two functionalities leads to robust signal detection, which is most desirable in practical UWA applications. MIMO linear equalizer (LE) is adopted to remove space-time interference, and a groupwise phase estimation and correction method is used to compensate the phase rotation. In addition, the layered space-time processing technology is adopted to enhance the equalization performance. The proposed equalization scheme is tested to be very robust with extensive experimental data collected at Kauai, Hawaii, in September 2005, and Saint Margaret's Bay, Nova Scotia, Canada, in May 2006.
\end{abstract}

\section{Introduction}

Underwater acoustic (UWA) channel is recognized as one of the most challenging channels in practical use [1]. The obstacles imposed by the water media on acoustic propagation are reflected in four aspects. First, the available channel bandwidth is very limited due to the frequencydependent attenuation. For example, in medium-range UWA communications, the bandwidth is on the order of a few tens of kilohertz. Second, the channel delay spread is very long due to the rich scattering environment. For instance, it could be over several tens of milliseconds (ms), leading to an equivalent discrete-time channel with several tens or even hundreds of channel taps in contrast to less than twenty taps in radio frequency (RF) communications. Third, the Doppler effect is very significant due to the low propagation speed of sound (about $1500 \mathrm{~m} / \mathrm{s}$ in water).
On one hand, the motion-induced Doppler shift causes a normalized carrier frequency offset (CFO) on the order of $10^{-4}$ to $10^{-3}$, compared to $10^{-8}$ to $10^{-6}$ in RF channels. On the other hand, the motion-induced waveform compression or dilation incurs nonnegligible symbol offset requiring signal resampling. Finally, the temporal variation of the UWA channel is very fast due to the dynamics of the water mass, which imposes difficulty on both channel estimation and phase tracking.

In the past three decades, significant progress has been achieved in UWA communications [2-14]. Earlier UWA communications adopted noncoherent frequency-shift keying (FSK) technology which enabled simple energybased signal detection combating the unpleasant effect of channel reverberation [2]. The drawback of FSK lies in its low transmission rate and also low bandwidth efficiency. The partial coherent modulation of differential phase shift 
keying (DPSK) was then chosen to achieve a bandwidth efficiency between noncoherent and fully coherent systems. It was until early 1990s, UWA transmission using the bandwidth-efficient coherent modulation appeared in [3]. Different from FSK, coherent transmission adopting modulations like phase shift keying (PSK) and quadrature amplitude modulation (QAM) requires proper cancelation of intersymbol interference (ISI) and compensation of phase distortion in signal detection; both tasks become very difficult under hash UWA channel conditions. Passivephase conjugation (PPC) [4] and time reversal (TR) [5] technologies, both having different principles from equalization, have been proposed to mitigate ISI. The detection using PPC technology, however, has poor performance when only a small number of receiving hydrophones are available [6]. As a result, equalization technology is more commonly adopted for coherent detection. Generally, equalization can be performed in either time-domain (TD) [711] or frequency-domain (FD) $[12-14]$. In $[7,8]$, the classic joint design of decision feedback equalizer (DFE) and phase-locked loop (PLL) has been proposed, and its iterative implementation can be found in [9]. In [10], by coupling PPC technology with a single-channel DFE, the correlation-based DFE is proposed and tested to be robust to different acoustic environments. The phase rotation is tracked with PLL technology. In [11], linear equalization combined with proper phase compensation has been proposed to minimize error propagation due to incorrect decision feedback. In [12], single-carrier frequency-domain equalization (SC-FDE) followed by phase compensation is proposed for single-carrier systems, and the frequencydomain equalization for multiple-carrier orthogonal frequency division multiplexing (OFDM) systems has been proposed in $[13,14]$. In [13], equalization is performed with adaptive channel estimation and phase tracking method. In [14], pilot-aided channel estimation is adopted for equalization, and a two-step Doppler compensation is adopted to remove phase rotation. While FD equalization enables low-complexity implementation even over highly dispersive channel, it usually requires extra guard intervals (GIs) among transmission blocks, which sacrifices the data transmission efficiency. Moreover, the inherent sensitivity of OFDM systems to carrier frequency offset makes robust signal detection very challenging especially with moving transceivers.

Despite the diverse equalization schemes, the demonstrated data rate of UWA communication is relatively low due to the natural limitation on the available channel bandwidth. In recent years, researchers have started to explore the spatial structure of the oceans to fundamentally improve the transmission rate. In the past years, MIMO UWA communications have been investigated in [15-19]. In [15], the number of the available degrees of freedom in the UWA channel is studied. In [16], coherent MIMO transmission has been presented, using a time reversal approach. In [17], the joint DFE and PLL scheme originally proposed in [7] for single input multiple output (SIMO) systems has been extended to MIMO cases. In [18], turbo linear equalization has been used for MIMO UWA communication. The FD equalization schemes proposed in $[12,14]$ have also been extended to MIMO systems in $[19,20]$, respectively.

In this paper, we propose a new time-domain MIMO equalization scheme for single carrier UWA communications. Different from conventional schemes performing joint equalization and phase tracking $[3,7-9,17]$, where the requirement for careful tuning of DFE and PLL parameters makes the system less stable [1], the new scheme decouples the interference-cancelation functionality and phasesynchronization functionality leading to a generalized equalizer structure consisting of an interference-cancelation (IC) equalizer and a phase compensator. MIMO linear equalizer (LE) is adopted to achieve low-complexity equalization and also to avoid the error propagation in DFE especially under harsh channel conditions. A novel groupwise phase estimation and correction method proposed in [21], which is insensitive to noise disturbance, is used to compensate phase rotations in the equalized symbols. The proposed equalization scheme has been adopted in a layered receiver structure for UWA communications and has been tested by high-rate MIMO experimental data measured off the northwestern coast of Kauai, Hawaii, in September 2005, and by both moving-source and fixed-source SIMO experimental data measured at Saint Margaret's Bay, Nova Scotia, Canada, in May 2006. We have achieved successful equalization in both experiments.

The rest of the paper is organized as follows. In Section 2, a general MIMO UWA system model is given. Based on the system model, MIMO channel estimation is introduced in Section 3, as the basis for equalizer design. The new MIMO equalization scheme is then developed in Section 4, where the MIMO IC equalization, the groupwise phase estimation and compensation, and the layered space-time processing technology are discussed, respectively. Section 5 reports the results on experimental data processing, and conclusion is drawn in Section 6.

\section{MIMO System Model}

For a MIMO UWA communication system employing $N$ transmitting transducers and $M$ receiving hydrophones, the baseband discrete-time signal received at the $m$ th hydrophone is represented as

$$
\tilde{y}_{m}(k)=\sum_{n=1}^{N} \sum_{l=0}^{L-1} \tilde{h}_{n, m}(k, l) \tilde{x}_{n}(k-l) e^{j \tilde{\phi}_{n, m}(k)}+\tilde{v}_{m}(k),
$$

where $\tilde{x}_{n}(k)$ is the effective transmission symbol of the $n$th transducer observed at the receiver, $\tilde{h}_{n, m}(k, l)$ and $\tilde{\phi}_{n, m}(k)$ are the $l$ th complex fading coefficient and the phase drift of the time-varying subchannel between the $n$th transducer and the $m$ th hydrophone, and $L$ is the channel length. The phase drift is a combining effect of the average Doppler shift, $\bar{f}_{n, m}$, the instantaneous Doppler, $f_{n, m}(k)$, and the coarse synchronization phase error, $\theta_{n, m}$, and can be expressed as $\widetilde{\phi}_{n, m}(k)=2 \pi\left[\bar{f}_{n, m}+f_{n, m}(k)\right] k T_{s}+\theta_{n, m}$, where $T_{s}$ is the symbol interval. The term $\tilde{v}_{m}(k)$ is the sample of a zeromean additive white Gaussian noise (AWGN) with power $\sigma_{v}^{2}$, on the $m$ th hydrophone. For practical UWA channels, the 
fading coefficient $\tilde{h}_{n, m}(k, l)$ usually changes slower than the instantaneous phase $\widetilde{\phi}_{n, m}(k)$; so it is appropriate to treat them separately [17].

The average Doppler shift $\bar{f}_{n, m}$ is caused by the relative motion between the transmitter and the receiver, while the instantaneous Doppler $f_{n, m}(k)$ modeled as a zero-mean time-varying random variable comes from the dynamics of the oceans. When the transmitter-receiver relative motion is nonnegligible, it causes the transmitted signal to be compressed or dilated at the receiver side $[22,23]$. In this case, the effective transmission symbol $\tilde{x}_{n}(k)$ in $(1)$ is not exactly the same as the originally transmitted symbol $x_{n}(k)$, where $x_{n}(k) \in \delta$ with $\&=\left\{\chi_{q}\right\}_{q=1}^{Q}$ being the modulation constellation of size $Q$. As a result, compensation of the average Doppler shift $\bar{f}_{n, m}$ and resampling of the received signal, herein named as Doppler preprocessing, are necessary before signal detection can be performed. An efficient average Doppler shift estimation method has been provided in [22]. After Doppler preprocessing, the signal model in (1) becomes

$$
y_{m}(k)=\sum_{n=1}^{N} \sum_{l=0}^{L-1} h_{n, m}(k, l) x_{n}(k-l) e^{j \phi_{n, m}(k)}+v_{m}(k),
$$

where $y_{m}(k), h_{n, m}(k, l), \phi_{n, m}(k)$, and $v_{m}(k)$ are the received symbol, the fading coefficient, the phase drift, and the additive noise after Doppler preprocessing, respectively. The phase term, $\phi_{n, m}(k)$, may contain residual Doppler shift effect due to nonideal Doppler shift estimation and compensation. The noise $v_{m}(k)$ is still zero-mean AWGN with variance power $\sigma_{v}^{2}$.

\section{Channel Estimation for MIMO UWA Communications}

As the basis for equalizer design, channel estimation for UWA communications is developed in this section. In training mode, MIMO channel estimation is performed with pilot symbols $\left\{p_{n}(k), 0 \leq k<N_{p}\right\}_{n=1}^{N}$ from all $N$ transducers. We assume that the time duration of the pilot sequences is less than the channel coherence time, in which case the fading coefficient $h_{n, m}(k, l)$ in (2) can be treated as approximately time-invariant, that is, $h_{n, m}(k, l) \approx h_{n, m}(l)$. The assumption is appropriate in many UWA communications. Then (2) can be represented in matrix form as

$$
\mathbf{y}_{m}=\sum_{n=1}^{N} \boldsymbol{\Phi}_{n, m} \mathbf{P}_{n} \mathbf{h}_{n, m}+\mathbf{v}_{m}
$$

where $\mathbf{y}_{m}=\left[y_{m}(L-1), y_{m}(L), \ldots, y_{m}\left(N_{p}-1\right)\right]^{t} \in \mathfrak{C}^{\left(N_{p}-L+1\right) \times 1}$ is the received sample vector at the $m$ th hydrophone with $(\cdot)^{t}$ denoting matrix transpose operation,

$$
\mathbf{P}_{n}=\left[\begin{array}{cccc}
p_{n}(L-1) & \cdots & p_{n}(1) & p_{n}(0) \\
p_{n}(L) & \cdots & p_{n}(2) & p_{n}(1) \\
\vdots & \ddots & \ddots & \vdots \\
p_{n}\left(N_{p}-1\right) & \cdots & p_{n}\left(N_{p}-L+1\right) & p_{n}\left(N_{p}-L\right)
\end{array}\right]
$$

is the matrix obtained with the $n$th pilot sequence, and $\boldsymbol{\Phi}_{n, m}=\operatorname{diag}\left\{e^{j \phi_{n, m}(L-1)}, e^{j \phi_{n, m}(L)}, \ldots, e^{j \phi_{n, m}\left(N_{p}-1\right)}\right\}, \mathbf{h}_{n, m}$ $=\left[h_{n, m}(0), h_{n, m}(1), \ldots, h_{n, m}(L-1)\right]^{t}$, and $\mathbf{v}_{m}=\left[v_{m}(L-\right.$ 1), $\left.v_{m}(L), \ldots, v_{m}\left(N_{p}-1\right)\right]^{t}$ are the phase drift matrix, the fading coefficient vector, and the noise vector, respectively. The operator $\operatorname{diag}\{\cdot\}$ returns a diagonal matrix. It is still difficult to estimate $\mathbf{h}_{n, m}$ and $\boldsymbol{\Phi}_{n, m}$ simultaneously from (3), due to the separation of $\boldsymbol{\Phi}_{n, m}$ and $\mathbf{h}_{n, m}$ by $\mathbf{P}_{n}$ on the righthand side. To proceed, we rewrite $\boldsymbol{\Phi}_{n, m}$ as

$$
\begin{aligned}
\Phi_{n, m}= & e^{j \phi_{n, m}(I)} \\
& \times \operatorname{diag}\left\{e^{j\left[\phi_{n, m}(L-1)-\phi_{n, m}(I)\right]}, e^{j\left[\phi_{n, m}(L)-\phi_{n, m}(I)\right]}, \ldots,\right. \\
& \left.e^{j\left[\phi_{n, m}\left(N_{p}-1\right)-\phi_{n, m}(I)\right]}\right\},
\end{aligned}
$$

where the index $I$ in $\phi_{n, m}(I)$ is determined as $I=\left\lceil\left(N_{p}+\right.\right.$ $L-2) / 2\rceil$ with $\lceil x\rceil$ denoting the smallest integer larger than $x$. The instantaneous phase drifts $\left\{\phi_{n, m}(k)\right\}_{k=L-1}^{N_{p}-1}$ in (5) could be significant. However, the differential phase drifts $\left\{\phi_{n, m}(k)-\phi_{n, m}(I)\right\}_{k=L-1}^{N_{p}-1}$ will be insignificant over a short period of time. Thus (5) can be approximated by $\boldsymbol{\Phi}_{n, m} \approx$ $e^{j \phi_{n, m}(I)} \mathbf{I}_{N_{p}-L+1}$ with $\mathbf{I}_{j}$ being an identity matrix of order $j$. Then (3) can be approximated by

$$
\begin{aligned}
\mathbf{y}_{m} & \approx \sum_{n=1}^{N} \mathbf{P}_{n}\left[e^{j \phi_{n, m}(I)} \mathbf{h}_{n, m}\right]+\mathbf{v}_{m} \\
& =\mathbf{P h}_{m}+\mathbf{v}_{m},
\end{aligned}
$$

where $\mathbf{P}=\left[\mathbf{P}_{1}, \mathbf{P}_{2}, \ldots, \mathbf{P}_{N}\right]$ and $\mathbf{h}_{m}=\left[e^{j \phi_{1, m}(I)} \mathbf{h}_{1, m}^{t}, e^{j \phi_{2, m}(I)} \mathbf{h}_{2, m}^{t}\right.$, $\left.\ldots, e^{j \phi_{N, m}(I)} \mathbf{h}_{N, m}^{t}\right]^{t}$. From (6), the minimum mean square error (MMSE) estimation of $\mathbf{h}_{m}$ is given by

$$
\widehat{\mathbf{h}}_{m}=\left(\mathbf{P}^{h} \mathbf{P}+\sigma_{v}^{2} \mathbf{I}_{N L}\right)^{-1} \mathbf{P}^{h} \mathbf{y}_{m},
$$

where $(\cdot)^{h}$ denotes matrix Hermitian transpose. The estimation in (7) is performed on all $M$ hydrophones to obtain MIMO channel estimation. It is noted that to guarantee the system equation (6) not to be underdetermined, a minimum of $N_{p} \geq(N+1) L-1$ pilot symbols are required for each of the $N$ transducers. Last, the same channel estimation procedure discussed above will also be adopted in the decision-directed mode, where the previously detected symbols instead of the pilot symbols are used for channel estimation.

\section{New Equalization Scheme for MIMO UWA Communication}

A new equalization scheme is discussed in this section, where the conventional equalization is first adopted to cancel the space-time interference among transmitted symbols, and then a novel phase estimation and compensation method is applied to remove the phase rotations in the equalized symbols. The layered receiver structure adopting the proposed equalization scheme is then demonstrated. 
4.1. MIMO Equalization for Space-Time Interference Cancelation. With the estimated MIMO channel, MIMO equalization can be performed. MIMO linear equalizer is adopted in this paper. In this case, the $k$ th equalized symbol of the $n$th transmission stream (from the $n$th transducer) is given by

$$
\hat{x}_{n}(k)=\sum_{m=1}^{M} \sum_{q=-K_{1}}^{K_{2}} c_{n, m}^{(q)} y_{m}(k-q),
$$

where $K_{1}, K_{2}$ are nonnegative integers, and $c_{n, m}^{(q)}$ denotes the $q$ th linear equalizer coefficient corresponding to the received sample of the $m$ th hydrophone for equalizing symbols of the $n$th stream. Without loss of generality, the same values of $K_{1}, K_{2}$ are used for all $(n, m)$ pairs, resulting in $N \times M \times\left(K_{1}+\right.$ $\left.K_{2}+1\right)$ equalizer taps in total.

Collecting the equalized symbols at time $k,\left\{\hat{x}_{n}(k)\right\}_{n=1}^{N}$, of all $N$ streams in a vector, leads to the matrix-form equalization model as

$$
\widehat{\mathbf{x}}(k)=\mathbf{C y},
$$

where $\widehat{\mathbf{x}}(k)=\left[\hat{x}_{1}(k), \hat{x}_{2}(k), \ldots, \hat{x}_{N}(k)\right]^{t} \in \mathrm{C}^{N \times 1}, \mathbf{y}=\left[\mathbf{y}^{t}(k-\right.$ $\left.\left.K_{2}\right), \mathbf{y}^{t}\left(k-K_{2}+1\right), \ldots, \mathbf{y}^{t}(k), \ldots, \mathbf{y}^{t}\left(k+K_{1}\right)\right]^{t} \in \mathrm{C}^{M\left(K_{1}+K_{2}+1\right) \times 1}$, and $\mathbf{C}=\left[\mathbf{c}^{\left(K_{2}\right)}, \mathbf{c}^{\left(K_{2}-1\right)}, \ldots, \mathbf{c}^{\left(-K_{1}\right)}\right] \in \mathrm{C}^{N \times M\left(K_{1}+K_{2}+1\right)}$ with $\mathbf{y}(k-$ $q) \in \mathcal{C}^{M \times 1}$ and $\mathbf{c}^{(q)} \in \mathcal{C}^{N \times M}$ defined, respectively, as

$$
\begin{gathered}
\mathbf{y}(k-q)=\left[y_{1}(k-q), y_{2}(k-q), \ldots, y_{M}(k-q)\right]^{t}, \\
\mathbf{c}^{(q)}=\left[\begin{array}{cccc}
c_{1,1}^{(q)} & c_{1,2}^{(q)} & \cdots & c_{1, M}^{(q)} \\
c_{2,1}^{(q)} & c_{2,2}^{(q)} & \cdots & c_{2, M}^{(q)} \\
\vdots & \vdots & \cdots & \vdots \\
c_{N, 1}^{(q)} & c_{N, 2}^{(q)} & \cdots & c_{N, M}^{(q)}
\end{array}\right] .
\end{gathered}
$$

To solve the equalizer matrix $\mathbf{C}$ in (9), the mean squared error (MSE) is applied leading to the cost function defined as follows:

$$
J(\mathbf{C})=\mathbb{E}\left[\|\mathbf{x}(k)-\hat{\mathbf{x}}(k)\|^{2}\right],
$$

where $\|\mathbf{a}\|$ is the Euclidean norm of vector $\mathbf{a}$, and $\mathbb{E}[\cdot]$ denotes mathematical expectation. By minimizing $J(\mathbf{C})$ in (11), the MMSE MIMO LE matrix is solved as

$$
\mathrm{C}_{\mathrm{MMSE}}=\mathbb{E}\left[\mathbf{x}(k) \mathbf{y}^{h}\right]\left\{\mathbb{E}\left[\mathbf{y} \mathbf{y}^{h}\right]\right\}^{-1}
$$

which is further simplified as

$$
\begin{aligned}
\mathbf{C}_{\text {MMSE }} & =\mathbf{R}^{h} \mathbf{H}^{h}\left(\mathbf{H H}^{h}+\frac{1}{\beta} \mathbf{I}_{M\left(K_{1}+K_{2}+1\right)}\right)^{-1} \\
& =\mathbf{R}^{h}\left(\mathbf{H}^{h} \mathbf{H}+\frac{1}{\beta} \mathbf{I}_{N\left(K_{1}+K_{2}+L\right)}\right)^{-1} \mathbf{H}^{h},
\end{aligned}
$$

where the derivation details are referred to the Appendix. In (13), the definitions of the channel matrix $\mathbf{H}$, the normalized transmission symbol correlation matrix $\mathbf{R}$, and the signal-tonoise ratio (SNR) $\beta$ are referred to (A.4), (A.6b), and (A.7), respectively. Since $\mathbf{R}$ is constant, the MIMO LE matrix only depends on the knowledge of MIMO channel and SNR.

Remark. In (13), two alternative solutions for the MIMO LE matrix $\mathbf{C}$ are provided. In the first solution (first equality), matrix inversion of order $M\left(K_{1}+K_{2}+1\right)$ is required. In the second solution, matrix inversion of order $N\left(K_{1}+K_{2}+L\right)$ is involved. Since matrix inversion is the main source of the computational complexity, the solution with smallerorder matrix inversion is always favored in practical MIMO systems.

With the designed LE matrix in (13), equalization can then be performed. Substituting (2) into (9), the $k$ th equalized symbol of the $n$th stream (or the $n$th element in $\widehat{\mathbf{x}}(k))$ is expressed as

$$
\begin{aligned}
& \hat{x}_{n}(k) \\
& =\sum_{m=1}^{M}\left[\sum_{q=-K_{1}}^{K_{2}} \sum_{i=1}^{N} \sum_{l=0}^{L-1} c_{n, m}^{(q)} h_{i, m}(k-q, l) x_{i}(k-q-l) e^{j \phi_{i, m}(k-q)}\right] \\
& \quad+\eta_{n}(k),
\end{aligned}
$$

where $\eta_{n}(k)=\sum_{m=1}^{M} \sum_{q=-K_{1}}^{K_{2}} c_{n, m}^{(q)} v_{m}(k-q)$ is the collection of additive noise $\left\{v_{m}(k)\right\}_{m=1}^{M}$ in the equalized symbol. As we can see from (14), the triple summation in the square bracket is the $m$ th hydrophone's contribution to the equalized symbol of the $n$th stream. Therefore, we have the following definition:

$$
\begin{aligned}
& \alpha_{n, m}(k) x_{n}(k)+\xi_{n, m}(k) \\
& \triangleq \sum_{q=-K_{1}}^{K_{2}} \sum_{i=1}^{N} \sum_{l=0}^{L-1} c_{n, m}^{(q)} h_{i, m}(k-q, l) x_{i}(k-q-l) e^{j \phi_{i, m}(k-q)},
\end{aligned}
$$

where $\alpha_{n, m}(k)$ denotes the scaling factor which is usually a complex value closely related to the equalizer taps $c_{n, m}^{(q)}$ and instantaneous phase rotation $e^{j \phi_{i, m}(k-q)}$, and $\xi_{n, m}(k)$ denotes residual interference from symbols other than $x_{n}(k)$. With above definition, (14) is simplified as

$$
\begin{aligned}
\hat{x}_{n}(k) & =\sum_{m=1}^{M} \alpha_{n, m}(k) x_{n}(k)+\xi_{n}(k)+\eta_{n}(k) \\
& =\left|\gamma_{n}(k)\right| e^{j \angle \gamma_{n}(k)} x_{n}(k)+\zeta_{n}(k),
\end{aligned}
$$

where $\gamma_{n}(k)=\sum_{m=1}^{M} \alpha_{n, m}(k)$ is the diversity combining gain of $M$ hydrophones, $\xi_{n}(k)=\sum_{m=1}^{M} \xi_{n, m}(k)$ is the overall residual interference, and $\zeta_{n}(k)=\xi_{n}(k)+\eta_{n}(k)$ is the effective noise consists of the residual interference and additive noise in the equalized symbol. Obviously, regardless of the effective noise, the equalized symbol $\hat{x}_{n}(k)$ in $(16)$ is an amplitude-scaled and phase-rotated version of the original 
symbol $x_{n}(k)$. The phase rotation $\angle \gamma_{n}(k)$ is a complicated term caused by phase drift $\phi_{n, m}(k)$. For systems employing coherent modulation schemes like phase shift keying (PSK), the phase rotation is hostile and must be compensated, which is the topic of the next subsection.

4.2. Groupwise Phase Estimation and Compensation. The groupwise phase estimation and correction method proposed in [21] is adopted to handle the phase rotations in the equalized symbol $\hat{x}_{n}(k)$, as shown in (16). The motivation for the groupwise phase estimation and correction method comes from the fact that the instantaneous phase drift $\phi_{n, m}(k)$ changes gradually while not arbitrarily from time to time due to the nature of ocean waters. In other words, the rotating phase $\angle \gamma_{n}(k)$ tends to be a constant over a small group of $N_{s}$ consecutive equalized symbols. The procedure for performing the groupwise phase estimation and compensation algorithm is presented in the following.

Initialization. For the equalized data block of size $N_{b}$, designate the first $N_{t s}$ symbols $\left\{x_{n}(k)\right\}_{k=1}^{N_{t s}}$ as the training symbols for phase reference and determine the initial phase $\psi_{n}(0)$ by

$$
\psi_{n}(0)=\frac{1}{N_{t s}} \sum_{k=1}^{N_{t s}}\left[\angle \hat{x}_{n}(k)-\angle x_{n}(k)\right]
$$

Then, partition the remaining $N_{b}-N_{t s}$ symbols into $N_{g}$ groups, each having $N_{s}$ symbols, except that the $N_{g}$ th group may have less than $N_{s}$ symbols. Further, for an $M$-ary PSK (MPSK) modulation constellation $\varsigma_{M}=$ $\{\exp [(j(m-1) 2 \pi) / M]\}_{m=1}^{M}$, define a phase quantization function $\mathbb{Q}[\cdot]$ as follows:

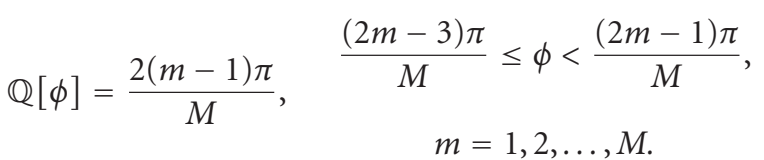

Set $g=1$.

Step 1. Compensate the phase of the gth group data by $e^{-j \psi_{n}(g-1)}$, yielding

$$
\begin{aligned}
& \hat{x}_{n}(g, k) \\
& \quad=\hat{x}_{n}\left(N_{t s}+(g-1) N_{s}+k\right) e^{-j \psi_{n}(g-1)}, \quad k=1,2, \ldots, N_{s} .
\end{aligned}
$$

Step 2. For each symbol in the $g$ th group, calculate its phase deviation from the corresponding nominal phase as

$$
\varphi_{n}(g, k)=\angle \hat{x}_{n}(g, k)-\mathbb{Q}\left[\angle \hat{x}_{n}(g, k)\right], \quad k=1,2, \ldots, N_{s} .
$$

Step 3. Calculate the average phase deviation and estimate the rotating phase, respectively, for the $g$ th group as

$$
\begin{gathered}
\Delta \psi_{n}(g)=\frac{1}{N_{s}} \sum_{k=1}^{N_{s}} \varphi_{n}(g, k), \\
\psi_{n}(g)=\psi_{n}(g-1)+\Delta \psi_{n}(g) .
\end{gathered}
$$

Step 4. Increment $g$ by 1 , repeat Steps $1-3$ till $g=N_{g}$.

After estimating the phases for the $N_{g}$ groups, we can compensate the phase rotation in the equalized symbols on group basis as

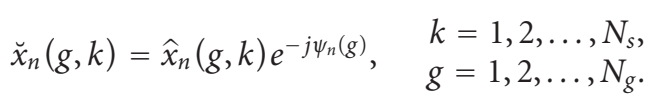

The phase-compensated symbol $\breve{x}_{n}(g, k)$ is then ready for detection. It is pointed out that if the last group has less than $N_{s}$ symbols, then the calculation through (19)-(23) needs to be carried out based on the actual number of symbols. Finally, the groupwise phase estimation method takes the advantage of insensitivity to noise perturbations, due to the averaging operation in (21).

4.3. Layered Space-Time Processing. Layered space-time processing was first proposed in [24], for frequency-flat fading channels. It was extended to frequency-selective fading channels in [25]. The basic idea of the layered time-space processing is to detect multiple data streams one by one in a specific order [26], so that the overall detection performance of all streams can be improved compared to joint detection.

In [25], the layered time-space processing is operated with the ordered successive interference cancelation (OSIC), where the transmitted streams are detected in an order that strong streams are detected earlier than weak streams, and each stream is processed with the interference from all previously detected streams already canceled out. For a MIMO UWA communication system using space-time trellis coding (STTC) [27], a layered receiver structure adopting the proposed equalization scheme discussed above is demonstrated in Figure 1, where $N$ stages each detecting one of the $N$ transmission streams are included. At each stage, channel equalization and phase compensation are operated separately.

The key for the success of OSIC-based space-time processing lies in the successive interference reconstruction and cancelation. For a detection order $\left\{o_{1}, o_{2}, \ldots, o_{N}\right\}$ with $o_{n}$ denoting the index of the stream detected at stage $n(1 \leq$ $n \leq N)$, the constructed interference of the $o_{n}$ th stream after it is detected is given as

$$
\widehat{I}_{o_{n}, m}(k)=\sum_{l=0}^{L-1} h_{o_{n}, m}(k, l) \bar{x}_{o_{n}}(k) \quad(1 \leq m \leq M),
$$

where $\bar{x}_{o_{n}}(k)=\tilde{x}_{o_{n}}(k) e^{j\left[\angle \hat{x}_{o_{n}}(k)-\angle \tilde{x}_{o_{n}}(k)\right]}$ with $\hat{x}_{o_{n}}(k)$ and $\tilde{x}_{o_{n}}(k)$ being the equalized symbol and the detected symbol of the $o_{n}$ th stream, respectively, as shown in Figure 1. 


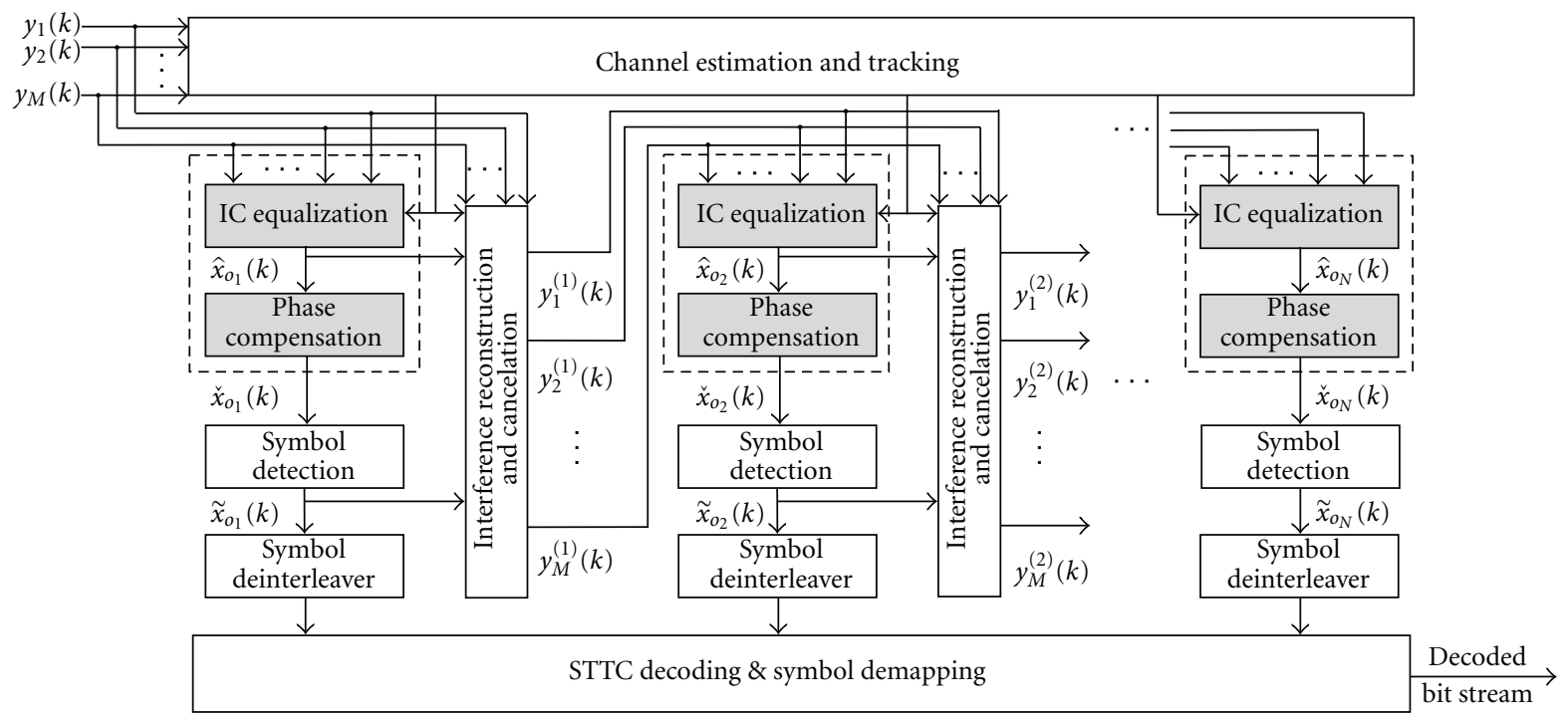

FIGURE 1: Layered MIMO receiver architecture adopting the proposed equalization (in each layer, an IC equalizer and a phase compensator perform interference cancelation and phase compensation separately).

The reconstructed interference of the $o_{n}$ th stream is then canceled out as

$$
y_{m}^{(n)}(k)=y_{m}^{(n-1)}(k)-\hat{I}_{o_{n}, m}(k), \quad(1 \leq m \leq M),
$$

where $y_{m}^{(n)}(k)$ denotes the received signal with the interference of the previous $n$ streams already subtracted out and is ready to be used for detecting the $o_{n+1}$ th stream. When detecting the $o_{1}$ th stream, we have $y_{m}^{(0)}(k)=y_{m}(k)$. The procedure in (24)-(25) is repeated until all streams are detected.

\section{Experimental Results}

The layered receiver structure adopting the proposed equalization scheme as shown in Figure 1 has been tested by two undersea experiments: Makai05 and Unet06. We present the details on data processing for both experiments in this section.

5.1. Results of Makai05 Experiment. Single-band (SB) and multiband (MB) MIMO underwater experiments were conducted off the northwestern coast of Kauai, Hawaii, in September 2005. In MB experiment, six signal bands each having a symbol rate of 2 kilo symbols per second (ksps) were used. The adoption of $\mathrm{MB}$ transmission aims to reduce the equivalent symbol-spaced channel length (thus reduce the equalization complexity), while still achieve a high data rate. Its spectral efficiency, however, is lower than SB transmission due to the insertion of guard bands among multiple signal bands. Signal detection for MB transmission has been presented in [17], and no results for SB transmission have been previously reported yet.

In this paper, we focus on SB experiment, which further includes low band (LB) transmission and high band (HB) transmission. In LB transmission, the carrier frequency was

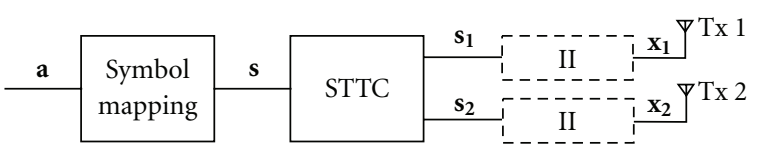

Figure 2: Signalling at the transmitter in Makai05 experiment.

$f_{c}=32 \mathrm{kHz}$ and the symbol interval was $0.1 \mathrm{~ms}$. The occupied channel bandwidth was $f_{b}=14 \mathrm{kHz}$ due to the use of a pulse shaping filter with roll-off factor 0.4 . In $\mathrm{HB}$ transmission, the carrier frequency was $f_{c}=37.5 \mathrm{kHz}$ and the symbol interval was $0.05 \mathrm{~ms}$. The occupied channel bandwidth was $f_{b}=25 \mathrm{kHz}$ due to a pulse shaping filter with roll-off factor 0.25. The modulations included BPSK, QPSK, and 8PSK for both LB and HB transmissions. The transmitter was a ten-transducer array with 2 meters separation between adjacent transducer elements and was deployed over the side of the Kilo Moana research vessel. The receiver consisted of eight hydrophones with 2 meters separation between adjacent hydrophone elements and was allowed to drift freely. The transmission range was 2 kilometers $(\mathrm{km})$.

In Figure 2, the signalling for a two-transducer transmission is shown. From the figure, the binary stream $\mathbf{a}$ is input to the symbol mapping module with the output modulation symbol stream denoted by $\mathbf{s}$. The symbol stream $\boldsymbol{s}$ is then encoded by space-time trellis coding, and the encoded stream is demultiplexed into two substreams $\boldsymbol{s}_{1}$ and $\boldsymbol{s}_{2}$ for two transducers. In this experiment, the four-state BPSK, fourstate QPSK, and eight-state 8PSK STTC codes provided in [27] were used, all for two-transducer transmission. Symbol interleavers $(\Pi)$ were optionally used to transform $\mathbf{s}_{1}$ and $\boldsymbol{s}_{2}$ into $\mathbf{x}_{1}$ and $\mathbf{x}_{2}\left(\mathbf{s}_{1}=\mathbf{x}_{1}\right.$ and $\mathbf{s}_{2}=\mathbf{x}_{2}$ when symbol interleaving was not applied). For the transmission with more than two transducers, the grouped STTC was used, with the details referred to [17]. 


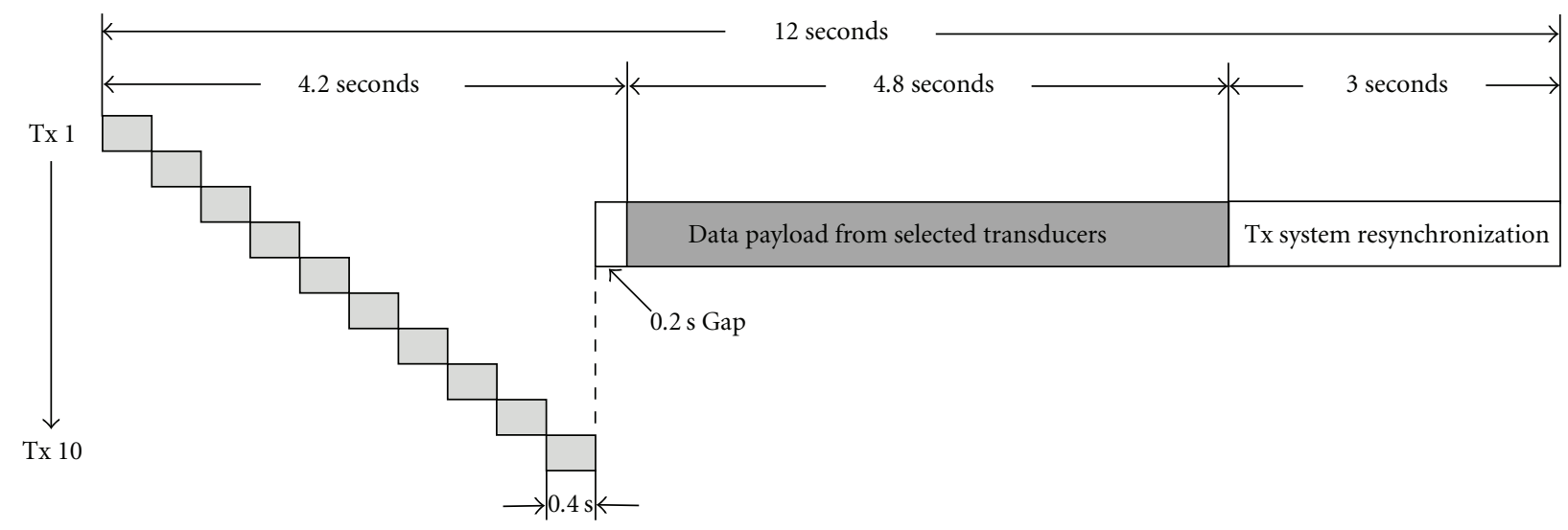

FIGURE 3: MIMO packet structure in Makai05 experiment.

The symbol stream was transmitted in packets. In Figure 3, the MIMO packet structure is shown. From the figure, the packet consists of three parts: the first part contains a sequence of probe signals transmitted by transducer one to transducer ten, plus a 0.2-second clear time. Each probe signal has a duration of 0.4 seconds and contains a linear frequency modulation (LFM) signal for synchronization. The second part contains data payload transmitted simultaneously by designated transducers depending on the experimental configuration. It has a time duration of 4.8 seconds. At the receiver side, part of the transmitted symbols will be used as pilot symbols for channel estimation, which will become evident shortly. The last part is a 3 -second clear time used for transmission system resynchronization. The whole packet has a time duration of 12 seconds.

The signal detection procedures for LB transmission and $\mathrm{HB}$ transmission are similar, and we present the details for LB transmission without loss of generality. As mentioned above, the LFM signal contained in the probe signal is used for packet synchronization due to its good correlation property. Moreover, the channel length $L$ can also be estimated with the LFM signals by measuring the span of the significant LFM correlation [11]. In Figure 4, an example of the normalized LFM correlation is depicted. It is clearly shown that there exists a correlation peak indicating the synchronization point. The significant correlation spans a range about $10 \mathrm{~ms}$, over which most of the channel energy is concentrated. The channel length is thus estimated as $L=100$, in terms of $L B$ symbol interval $T_{s}=0.1 \mathrm{~ms}$.

Once the packet synchronization was achieved, the MIMO channel was initially estimated using pilot symbols located at the front of the data payload with the method described in Section 3. For the channel length of $L=$ 100 requiring a minimum training length of 299 with two-transducer MIMO transmission, a larger pilot length $N_{p}=600$ was selected so as to obtain an accurate channel estimation and also mitigate the effect of additive noise. Such choice of pilot length corresponds to a time duration of $60 \mathrm{~ms}$, which is less than the channel coherence time about $200 \mathrm{~ms}$ corresponding to a maximum Doppler spread between $2 \sim 3 \mathrm{~Hz}$ in this experiment. The approximation of

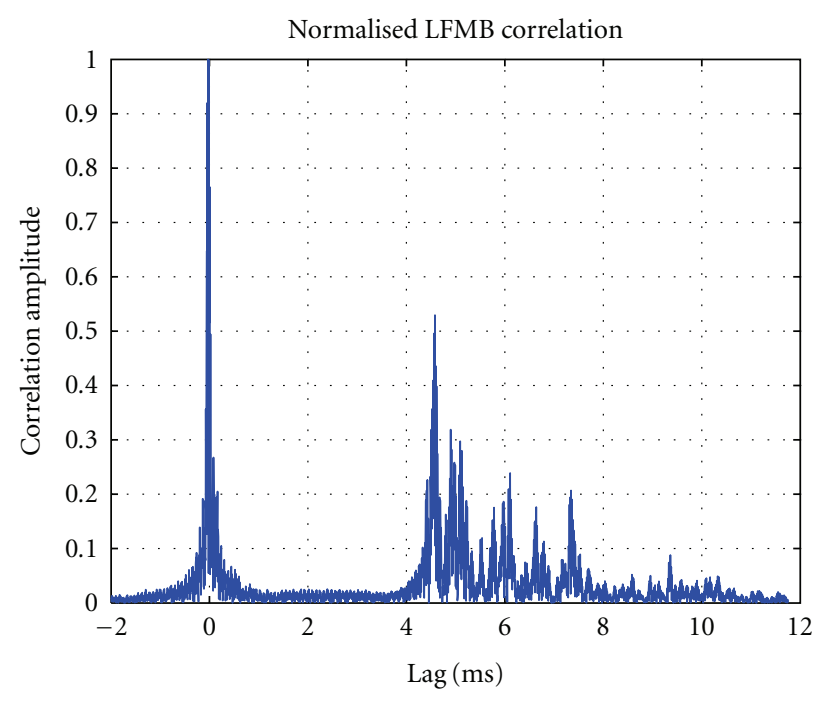

FIgURE 4: An example of normalized LFM correlation.

phase drift in (6) is also appropriate in this case, since the maximum possible differential phase drift is less than $\pi / 8$. Figures 5 and 6 show two examples of the estimated channel impulse responses (CIRs) for the two-transducer eighthydrophone MIMO transmissions with BPSK and QPSK modulations, respectively. Obviously, all subchannels are sparse with two distinct peaks over the depicted delay spread. The estimated MIMO channel with 8PSK modulation was similar to that depicted in Figure 6 and is not shown for brevity. The estimated channel was also used to determine the detection order in the layered space-time processing. For each stream (transducer), its related channel energy is defined as the summation of all related subchannel energies. Then a simple ordering criterion is to compare the related channel energies among streams, and the stream having larger related channel energy is detected earlier.

Periodic pilot sequences were inserted to divide the data payload into frames, and each frame was artificially partitioned into blocks for processing, as shown in Figure 7. We chose the block size $N_{b}=200$, corresponding to $20 \mathrm{~ms}$. 


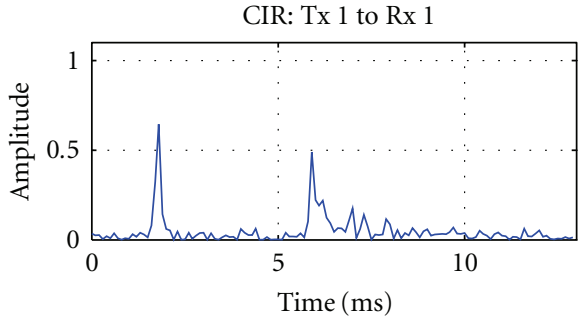

(a)

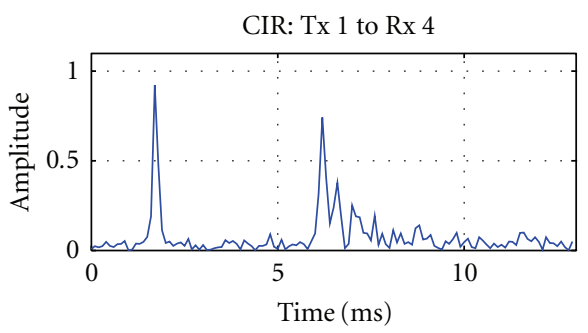

(c)

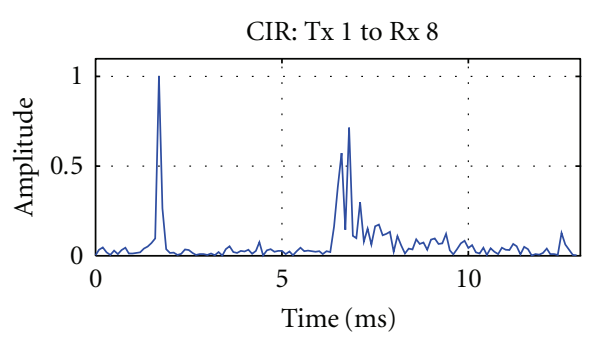

(e)

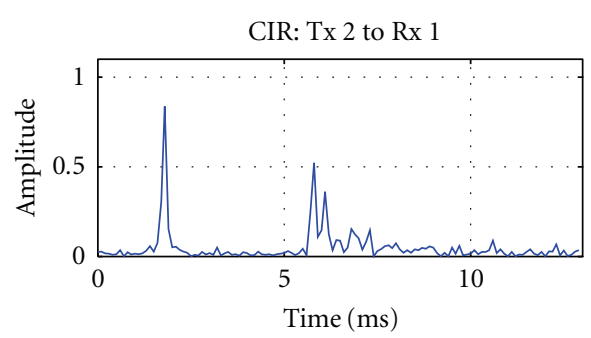

(b)

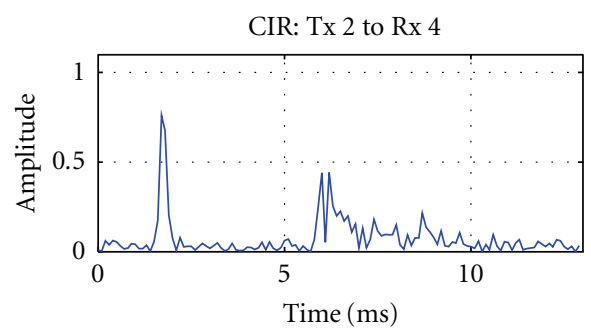

(d)

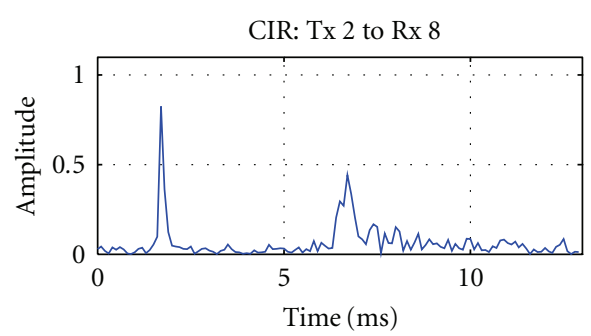

(f)

FIGURE 5: Estimated channel impulse responses for two-transducer eight-hydrophone transmission with BPSK modulation.

The initial MIMO channel estimation was employed to detect the block following the first pilot sequence, using the layered detection scheme. In each layer, $N_{b}$ symbols of the corresponding stream were first equalized. The LE parameters $K_{1}$ and $K_{2}$ in (8) were selected as $K_{1}=K_{2}=$ $L-1$. An interesting observation was that using smaller $K_{2}<L$ only slightly degraded the equalization performance while decreased the computational complexity considerably. The $N_{b}$ equalized symbols were then fed into the phase compensation unit, as shown in Figure 1. In the operation of groupwise phase estimation and correction, the group size $N_{s}=20$ was used, and there were $N_{g}=10$ groups in one block. After phase compensation, the $N_{b}$ symbols were detected. The detected symbols together with the equalized symbols were also used to construct the interference of the detected stream, which was subtracted out of the received signal for detecting the next stream. The block processing was finished when all $N$ streams were detected. To effectively track the time variation of MIMO channel, $N_{p}$ previously detected symbols (part of them could be pilot symbols) of all streams were used to reestimate the channel, as shown in Figure 7. The updated channel was then used to detect the current data block in a similar way mentioned above. The channel reestimation and detection procedure continued until the next pilot sequence started. The insertion of periodic pilot sequences aimed to combat possible error propagation of decision-directed channel reestimation, and the new frame was processed in the same way as the previous one. The frame size was selected to achieve a tradeoff between detection performance and training overhead. In the processing, we selected $N_{f}=16,000$ which incurred only $5 \%$ overall training overhead, comparing to $20 \%$ training overhead with conventional algorithms $[17,28]$. Once an entire packet was detected, the $N$ symbol streams were deinterleaved (if the symbol interleaver was used) and then sent to the STTC decoding and symbol demapping module to produce the decoded information bit stream, as shown in Figure 1. The STTC decoding was easily implemented with Viterbi algorithm.

In Figure 8, the sequence of estimated CIRs obtained during packet detection is depicted, where the temporal variations of the four subchannels are clearly shown over the depicted time interval within one packet. In Figure 9, the scatter plot shows two channels (hydrophone one and hydrophone eight) of received baseband signal and the equalized and phase-corrected BPSK symbols of transducer one for a $2 \times 8 \mathrm{MIMO}$ packet. In Figure 9(c), the phase rotations in the equalized symbols are obvious when comparing with the phase-corrected symbols shown in Figure 9(d). The phase-corrected symbols have been properly classified into 


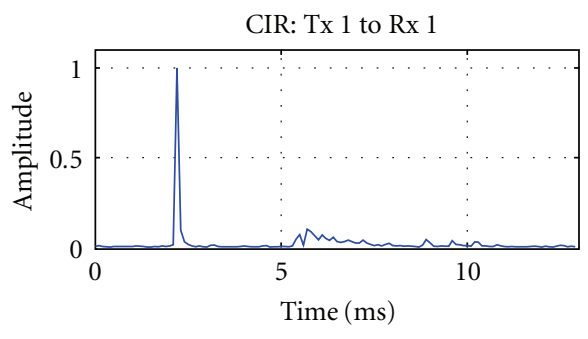

(a)

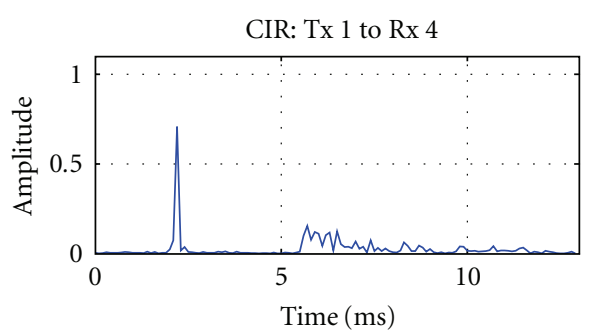

(c)

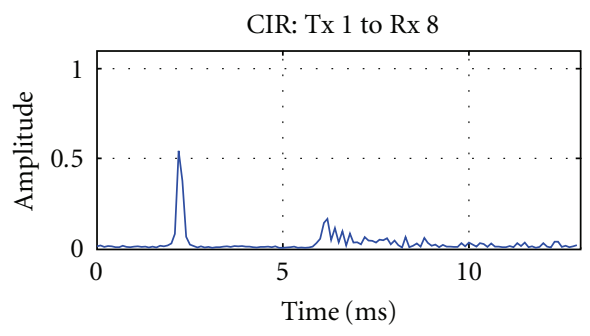

(e)

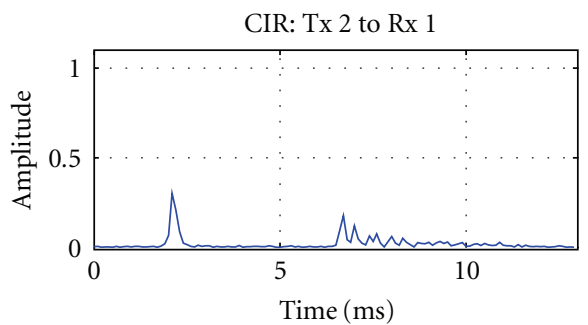

(b)

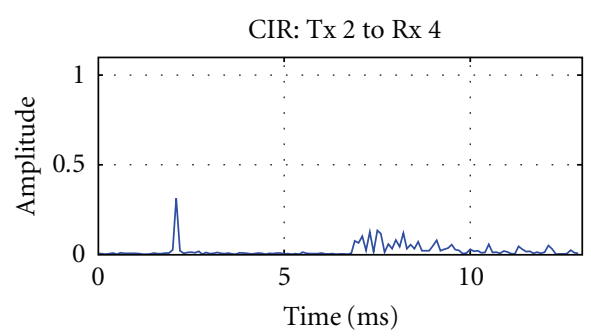

(d)

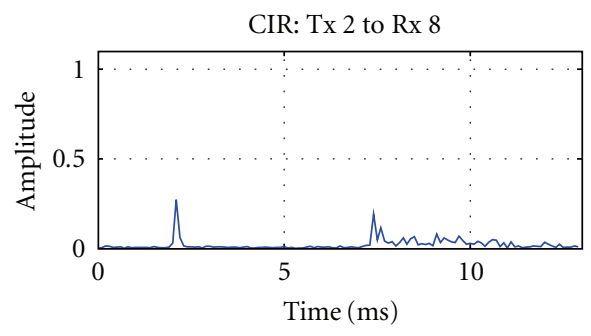

(f)

FIGURE 6: Estimated channel impulse responses for two-transducer eight-hydrophone transmission with QPSK modulation.

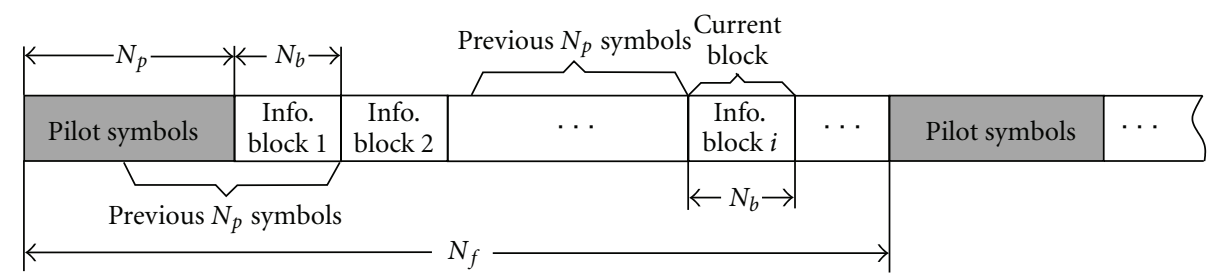

Figure 7: Partition of the transmitted data payload for block detection at the receiver (the same structure applied to all $N$ transmission streams).

TABLE 1: BER of LB $2 \times 8$ MIMO with BPSK Modulation.

\begin{tabular}{|c|c|c|c|c|}
\hline Packet index & $\begin{array}{c}\text { Uncoded BER of } \\
\operatorname{Tx} 1 \\
\end{array}$ & $\begin{array}{l}\text { Uncoded BER of } \\
\operatorname{Tx} 2 \\
\end{array}$ & $\begin{array}{l}\text { Uncoded BER of } \\
\text { Tx } 1 \text { and } 2\end{array}$ & $\begin{array}{c}\text { BER after STTC } \\
\text { decoding }\end{array}$ \\
\hline 1 & $2.800 \mathrm{e}-3$ & $4.178 \mathrm{e}-3$ & $3.489 \mathrm{e}-3$ & $2.000 \mathrm{e}-4$ \\
\hline 2 & $4.237 \mathrm{e}-4$ & $1.695 \mathrm{e}-4$ & $2.966 \mathrm{e}-4$ & 0 \\
\hline 3 & $1.556 \mathrm{e}-3$ & $9.333 \mathrm{e}-4$ & $1.244 \mathrm{e}-3$ & $1.111 \mathrm{e}-4$ \\
\hline 4 & $1.316 \mathrm{e}-4$ & $5.482 \mathrm{e}-4$ & $3.399 \mathrm{e}-4$ & 0 \\
\hline 5 & $2.412 \mathrm{e}-4$ & $1.096 \mathrm{e}-4$ & $1.695 \mathrm{e}-4$ & $6.579 \mathrm{e}-5$ \\
\hline 6 & $4.240 \mathrm{e}-5$ & $1.271 \mathrm{e}-4$ & $8.475 \mathrm{e}-5$ & 0 \\
\hline 7 & $8.114 \mathrm{e}-4$ & $5.044 \mathrm{e}-4$ & $6.579 \mathrm{e}-4$ & $6.579 \mathrm{e}-5$ \\
\hline 8 & $1.059 \mathrm{e}-4$ & $6.360 e-5$ & $8.475 e-5$ & 0 \\
\hline Mean & $7.645 e-4$ & $8.294 \mathrm{e}-4$ & $7.954 \mathrm{e}-4$ & $5.533 e-5$ \\
\hline
\end{tabular}




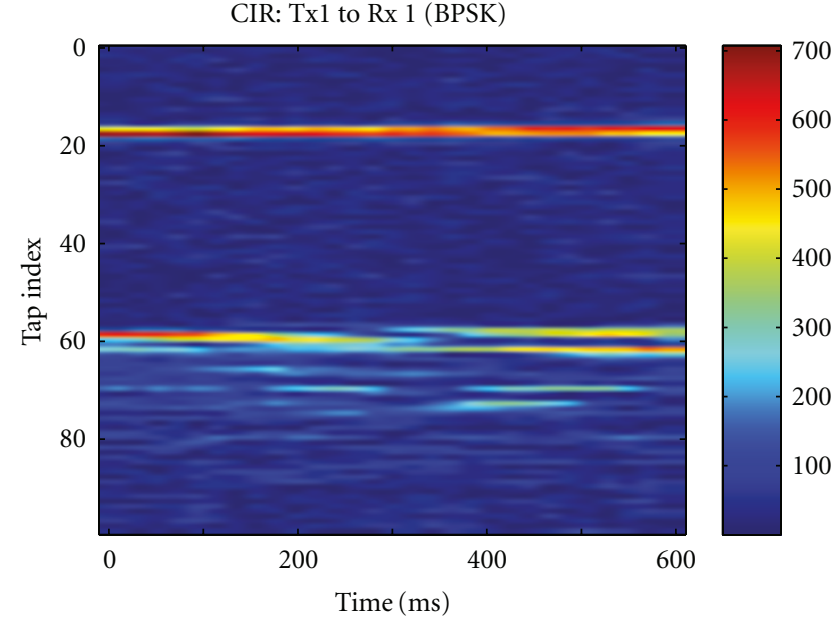

(a)

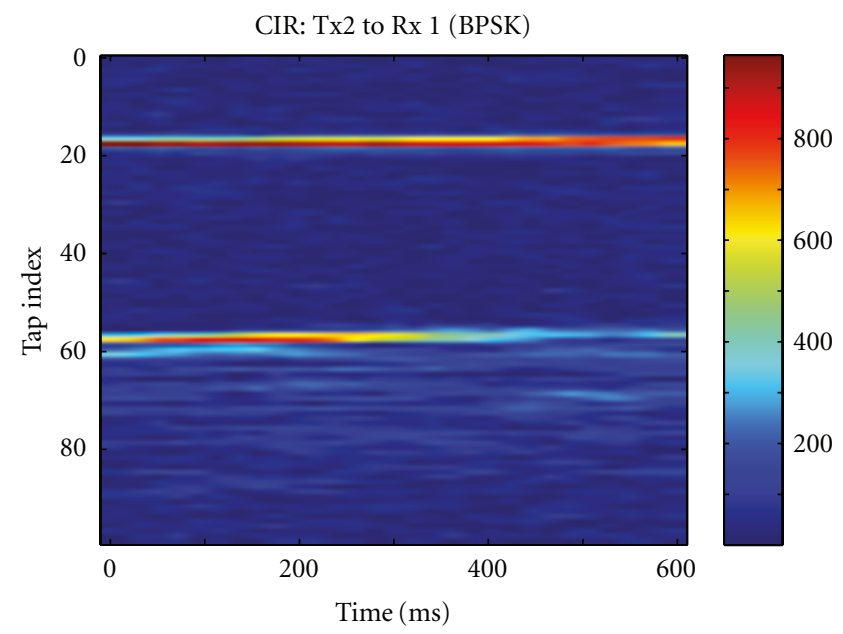

(c)

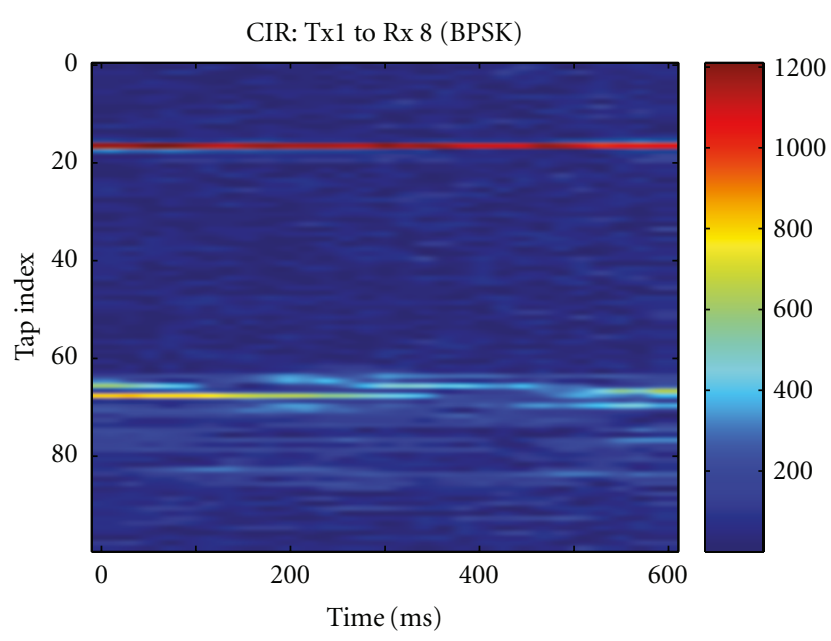

(b)

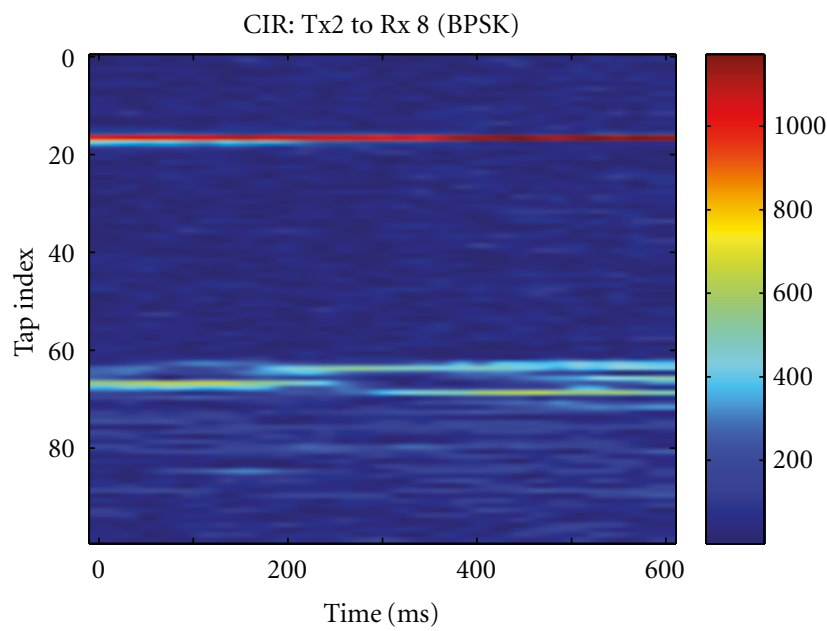

(d)

FIGURE 8: Demonstration of channel time variation within one packet (BPSK modulation).

TABLE 2: BER of LB $2 \times 8$ MIMO with QPSK Modulation.

\begin{tabular}{|c|c|c|c|c|}
\hline Packet index & $\begin{array}{c}\text { Uncoded BER of } \\
\text { Tx } 1\end{array}$ & $\begin{array}{c}\text { Uncoded BER of } \\
\operatorname{Tx} 2\end{array}$ & $\begin{array}{c}\text { Uncoded BER of } \\
\text { Tx } 1 \text { and } 2\end{array}$ & $\begin{array}{c}\text { BER after STTC } \\
\text { decoding }\end{array}$ \\
\hline 1 & $1.656 \mathrm{e}-3$ & $1.756 \mathrm{e}-1$ & $8.863 e-2$ & $6.984 \mathrm{e}-2$ \\
\hline 2 & $1.126 \mathrm{e}-4$ & $1.707 \mathrm{e}-2$ & $8.592 \mathrm{e}-3$ & $1.374 \mathrm{e}-3$ \\
\hline 3 & $1.351 \mathrm{e}-4$ & $1.498 \mathrm{e}-3$ & $8.164 \mathrm{e}-4$ & $2.252 \mathrm{e}-5$ \\
\hline Mean & $6.346 \mathrm{e}-4$ & $6.472 \mathrm{e}-2$ & $3.268 \mathrm{e}-2$ & $2.375 \mathrm{e}-2$ \\
\hline
\end{tabular}

TABLE 3: BER of LB $2 \times 8$ MIMO with 8PSK Modulation.

\begin{tabular}{lcccc}
\hline Packet index & Uncoded BER of & Uncoded BER of & Uncoded BER of & BER after STTC \\
dx 1 and 2 & Tx 2 & $8.481 \mathrm{e}-2$ & $2.508 \mathrm{e}-2$ \\
1 & Tx 1 & $1.614 \mathrm{e}-1$ & $6.831 \mathrm{e}-2$ & $1.265 \mathrm{e}-2$ \\
2 & $8.213 \mathrm{e}-3$ & $1.199 \mathrm{e}-1$ & $7.656 \mathrm{e}-2$ & $1.887 \mathrm{e}-2$ \\
\hline
\end{tabular}




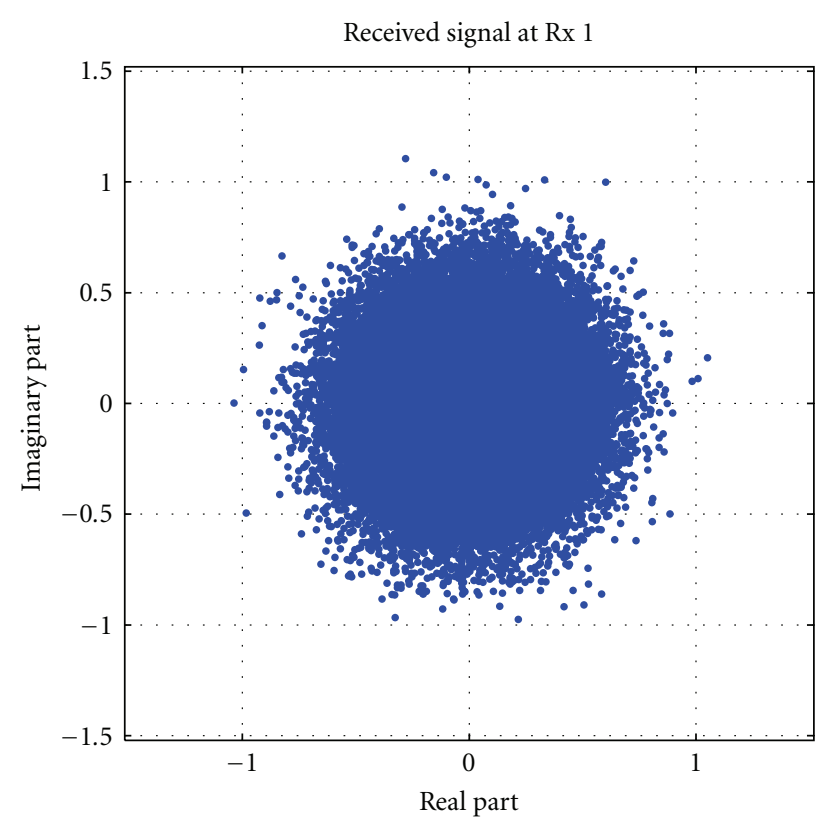

(a)

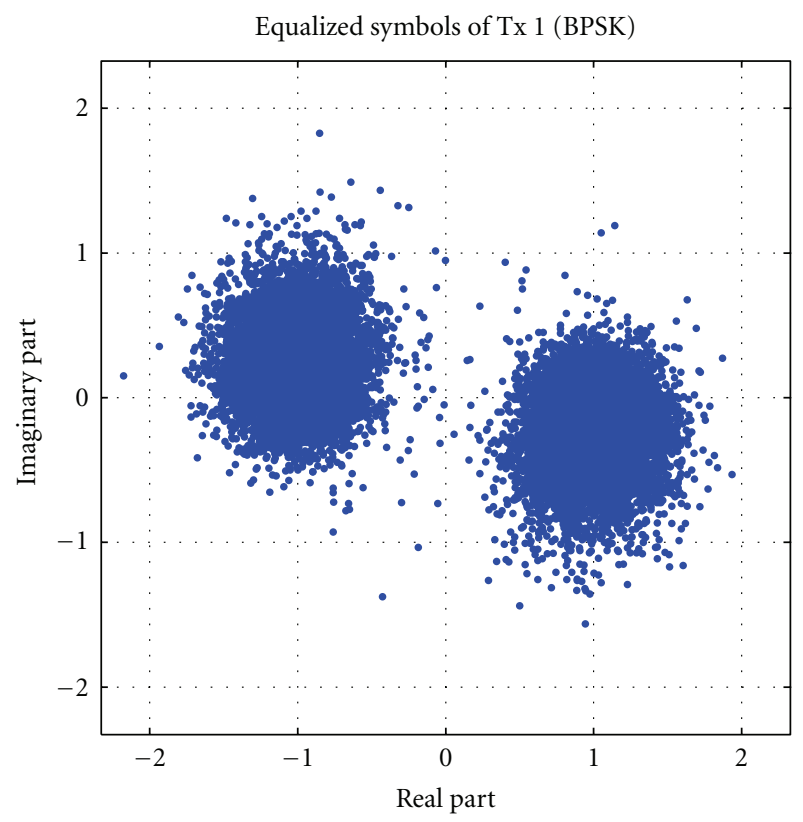

(c)

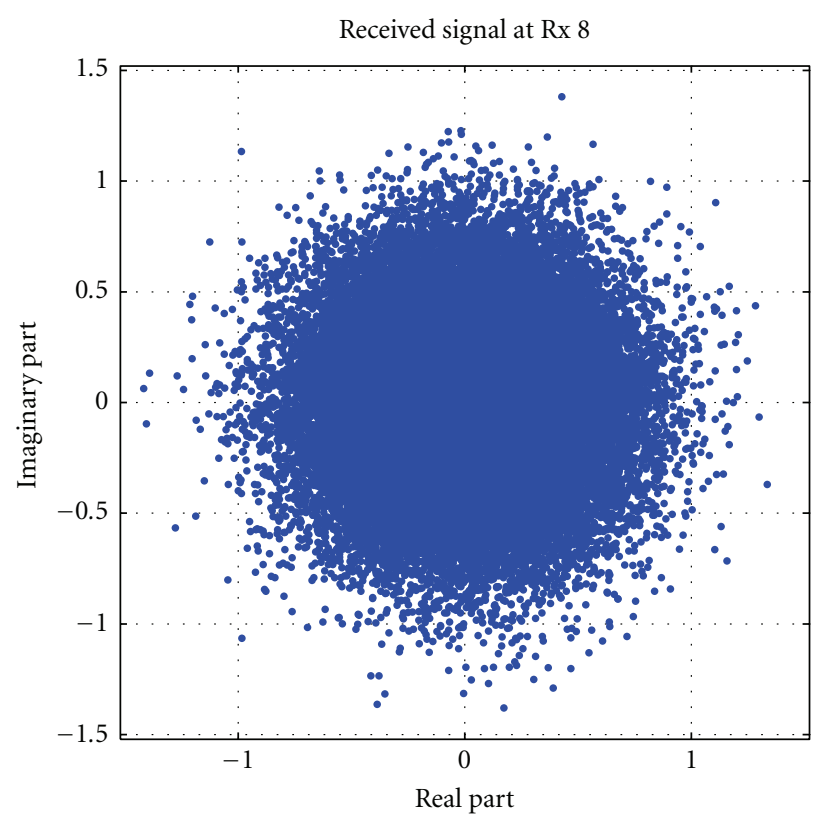

(b)

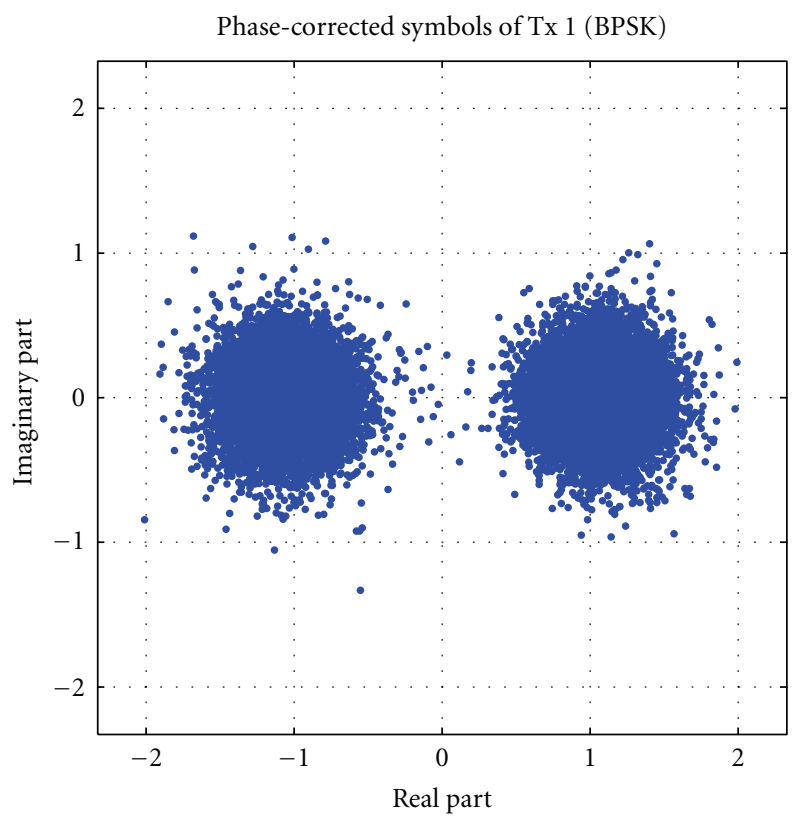

(d)

FIGURE 9: Received baseband signal, equalized and phase-corrected BPSK symbols.

two clusters centered at the two nominal BPSK modulation symbols -1 and +1 . Similar observations are found with the equalized and phase-corrected QPSK and 8PSK symbols, as depicted in Figure 10. All demonstrations have therefore verified the necessity and effectiveness of the phase compensation operation.

From the available experimental data, we had eight $2 \times 8$ BPSK packets, three $2 \times 8$ QPSK packets, and two $2 \times 88$ PSK packets for LB transmission. We processed all these packets with the proposed detection scheme, and the bit error rate
(BER) results for the $2 \times 8 \mathrm{MIMO}$ transmission with BPSK, QPSK, and 8PSK modulations are listed in Table 1 through, Table 3. It is noted that symbol interleaving was applied only to even-indexed packets in all three tables. From these tables, we make three observations. First, the BER performance degrades when the size of the constellation size increases, by comparing the average uncoded BERs listed in the last lines of the three tables. Second, the average uncoded BERs of the two streams are comparable with BPSK modulation, while the first stream has better average uncoded BER than 


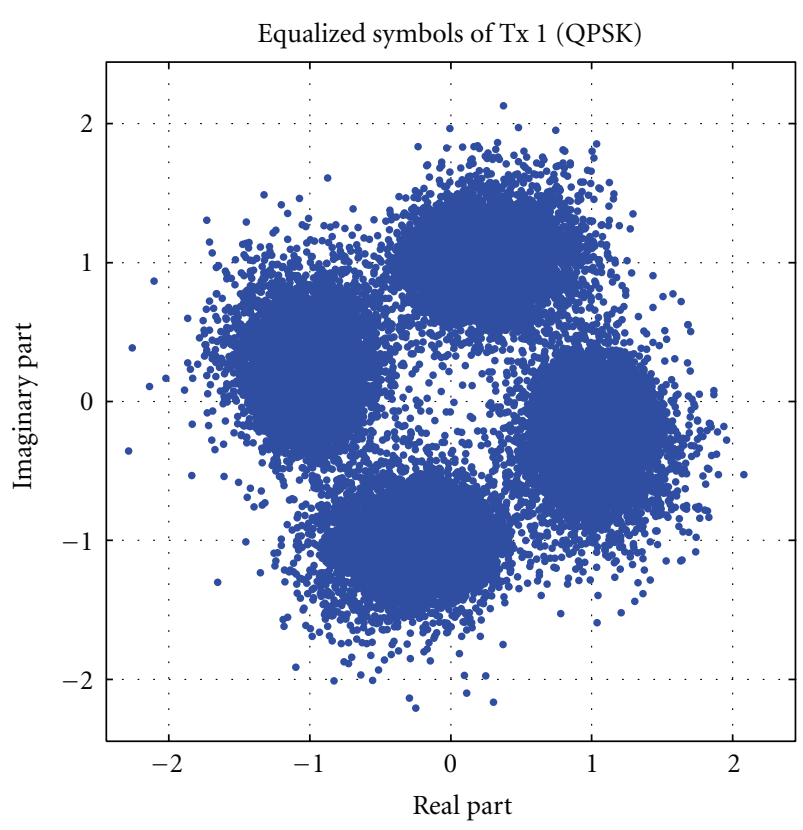

(a)

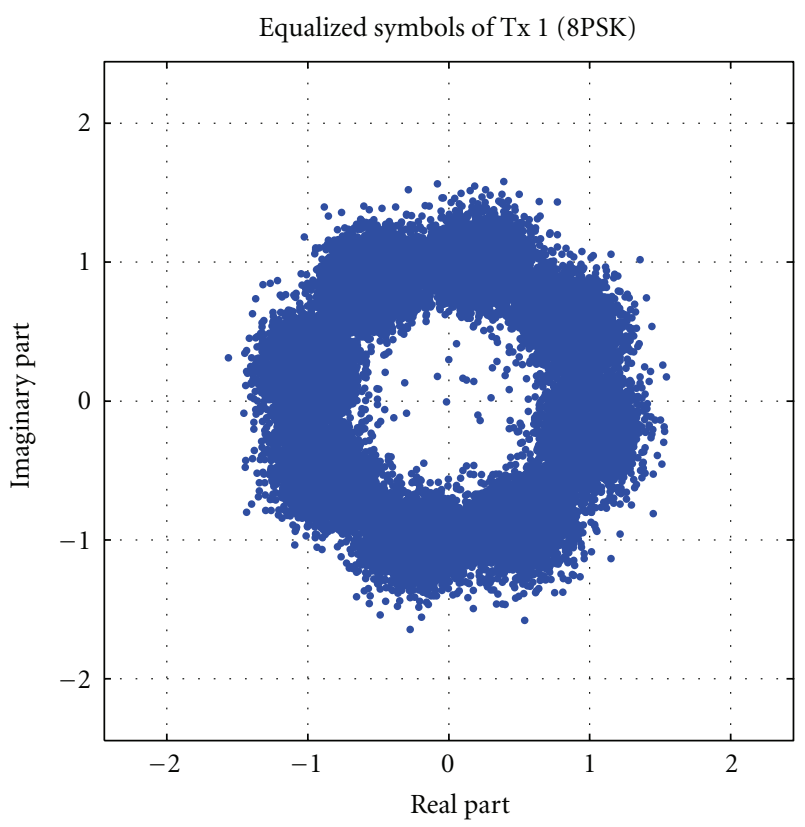

(c)

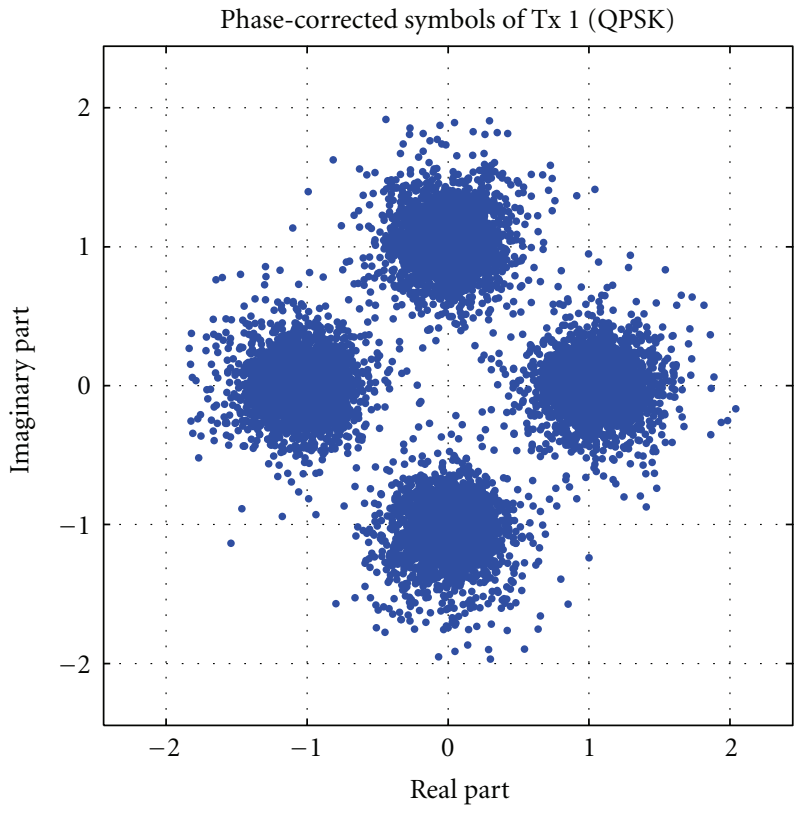

(b)

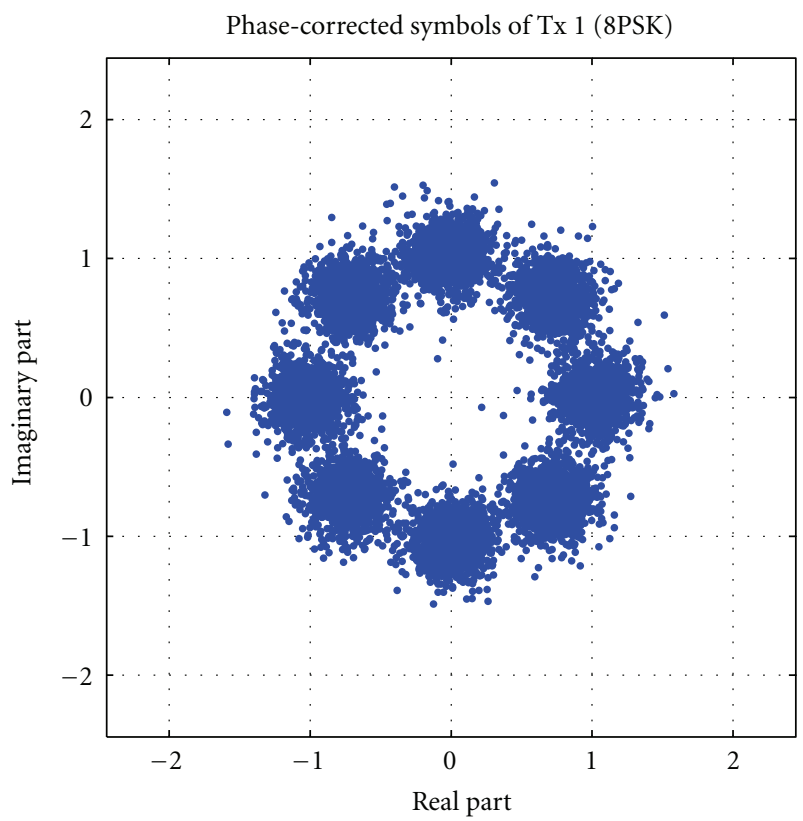

(d)

FIgURE 10: Equalized and phase-corrected QPSK and 8PSK symbols.

TABLE 4: BER of HB $2 \times 8$ MIMO with BPSK Modulation.

\begin{tabular}{|c|c|c|c|c|}
\hline Packet index & $\begin{array}{l}\text { Uncoded BER of } \\
\text { Tx } 1\end{array}$ & $\begin{array}{l}\text { Uncoded BER of } \\
\operatorname{Tx} 2\end{array}$ & $\begin{array}{c}\text { Uncoded BER of } \\
\text { Tx } 1 \text { and } 2\end{array}$ & $\begin{array}{c}\text { BER after STTC } \\
\text { decoding }\end{array}$ \\
\hline 1 & $1.190 \mathrm{e}-4$ & $1.212 \mathrm{e}-3$ & $6.656 \mathrm{e}-4$ & $1.082 \mathrm{e}-4$ \\
\hline 2 & $9.740 e-5$ & $7.359 \mathrm{e}-4$ & $4.167 e-4$ & 0 \\
\hline 3 & $1.840 \mathrm{e}-4$ & $1.190 \mathrm{e}-3$ & $6.872 \mathrm{e}-4$ & $7.576 \mathrm{e}-5$ \\
\hline 4 & $7.325 \mathrm{e}-3$ & $7.993 \mathrm{e}-3$ & $7.659 \mathrm{e}-3$ & $1.064 \mathrm{e}-3$ \\
\hline 5 & $6.494 \mathrm{e}-5$ & $1.027 \mathrm{e}-2$ & $5.168 \mathrm{e}-3$ & $4.361 \mathrm{e}-3$ \\
\hline 6 & $1.447 \mathrm{e}-2$ & $2.103 e-2$ & $1.775 \mathrm{e}-2$ & $2.597 \mathrm{e}-4$ \\
\hline Mean & $3.710 \mathrm{e}-3$ & $7.072 \mathrm{e}-3$ & $5.391 \mathrm{e}-3$ & $9.781 \mathrm{e}-4$ \\
\hline
\end{tabular}




\begin{tabular}{|l|c|c|c|c|c|c|}
\hline LFMB & Gap & $\begin{array}{c}\text { m-seq. } \\
(1023)\end{array}$ & Gap & $\begin{array}{c}\text { Data payload } \\
(40397)\end{array}$ & Gap & LFME \\
\hline \multicolumn{5}{|c|}{$\longrightarrow$} \\
\hline
\end{tabular}

FIGURE 11: SIMO packet structure in Unet06 experiment.

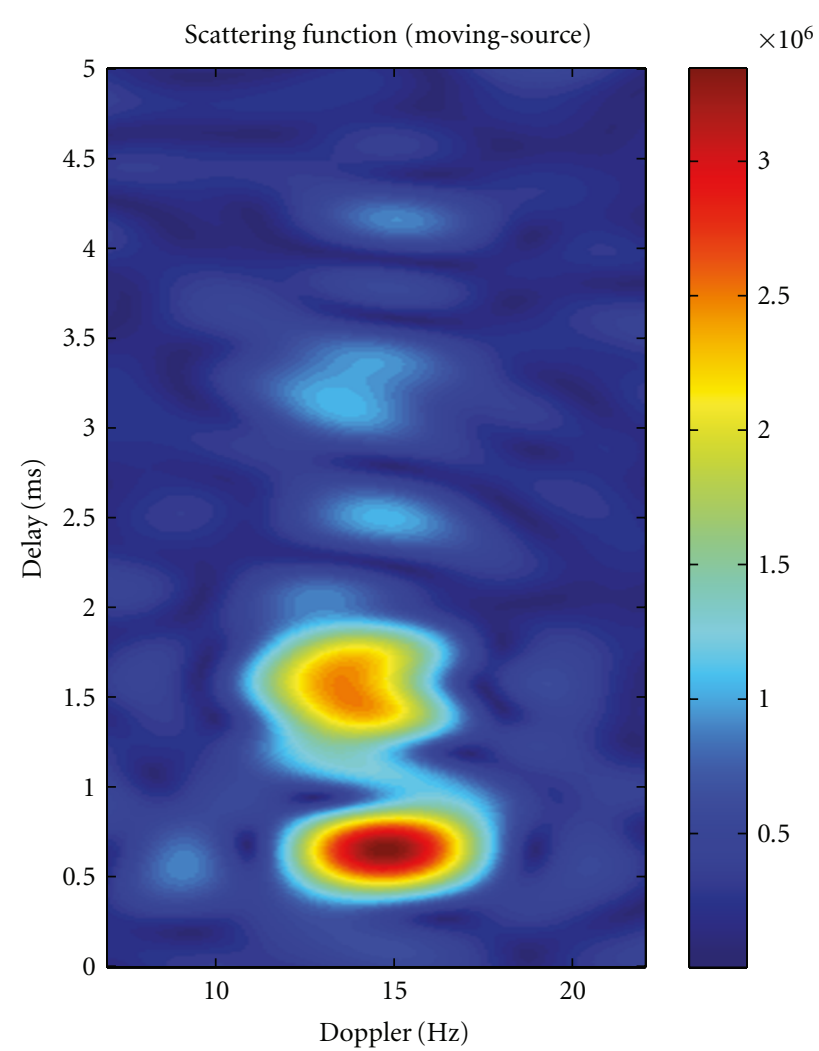

(a)

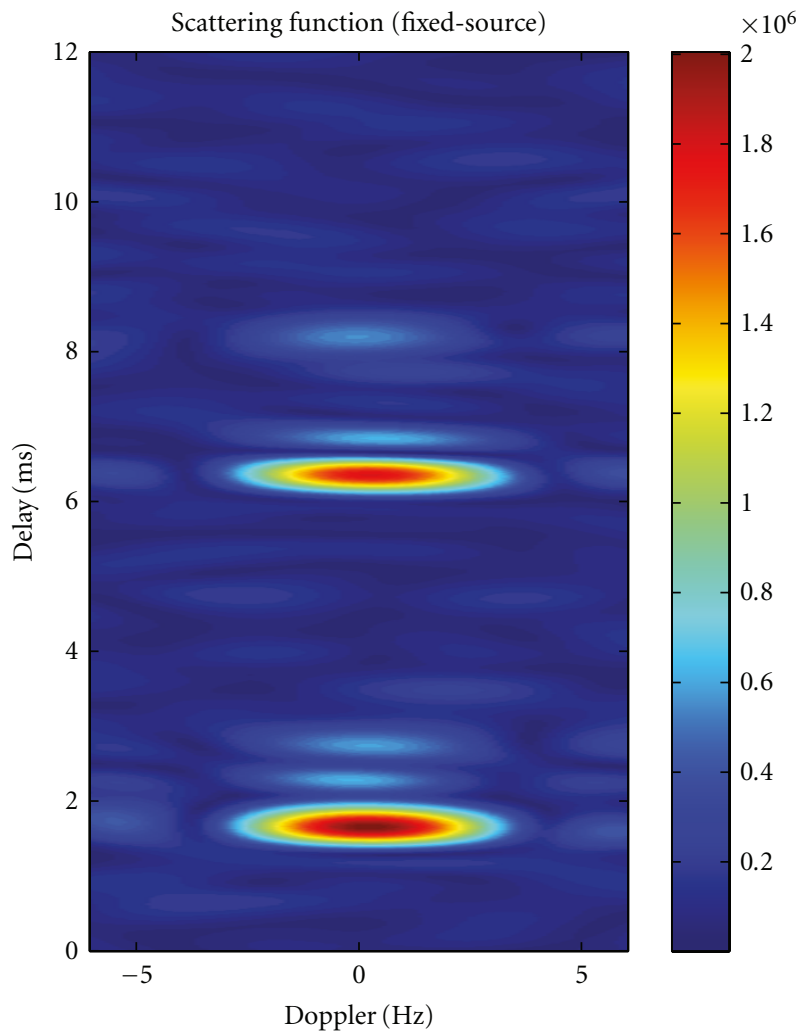

(b)

FIgURE 12: Channel scattering functions.

that of the second stream with QPSK and 8PSK modulations. This phenomenon is well explained by the fact that the two streams have similar related channel energy in BPSK case as shown in Figure 5. In contrast, stream one has a larger related channel energy than stream two in QPSK case, as shown in Figure 6. Third, the BER performance of all packets is improved with STTC decoding. The even-indexed packets have more significant improvement after STTC decoding than the odd-indexed packets, due to the use of symbol interleaving.

We finally presented the results for $\mathrm{HB}$ transmission. The channel length was measured as $L=150$ compared to $L=$ 100 with LB transmission, due to the smaller symbol interval in this case. The BER results for HB transmission are shown in Table 4 with BPSK modulation. The average uncoded BER is on the order of $5 \times 10^{-3}$, which is not as good as that of Table 1 due to the increased transmission rate.

5.2. Results of Unet06 Experiment. SIMO UWA communication experiments with both moving-source transmission and fixed-source transmission were conducted at Saint Margaret's Bay, Nova Scotia, Canada, in May 2006. For moving-source transmission, the transmitter was deployed 21 meters deep in the water and towed at a speed up to 4 knots. The communication distance was ranging from $1 \mathrm{~km}$ to $3 \mathrm{~km}$. For fixed-source transmission, the transmitter was suspended in water at 21 meters depth and 44 meters above the sea bottom, and the transmission range was fixed as $3.06 \mathrm{~km}$. The receiver consisted of eight hydrophones arranged unequally on a 1.86-meter vertical array, which was suspended 30 meters deep in the water. QPSK modulation was used with a symbol rate of $4 \mathrm{ksps}$. The carrier frequency was $f_{c}=17 \mathrm{kHz}$.

The structure of the transmission packet is depicted in Figure 11. It starts with an LFM signal named LFMB and ends with another LFM signal called LFME. An $m$-sequence of length 1023 is embedded in the packet, and the data payload carries 40397 symbols. The whole packet has a time duration of 15 seconds.

Without loss of generality, the processing details on moving-source packets are presented. As before, packet 


\begin{tabular}{|c|c|c|c|c|c|}
\hline$\leftarrow N_{p} \rightarrow$ & & & $\begin{array}{l}\text { Previous } \\
N_{p} \text { symbols }\end{array}$ & $\underbrace{\begin{array}{c}\text { Current } \\
\text { block }\end{array}}$ & \\
\hline $\begin{array}{c}\text { Pilot } \\
\text { symbols }\end{array}$ & $\begin{array}{l}\text { Info. } \\
\text { block } 1\end{array}$ & $\begin{array}{l}\text { Info. } \\
\text { block } 2\end{array}$ & $\cdots$ & $\begin{array}{l}\text { Info. } \\
\text { block } i\end{array}$ & $\begin{array}{c}\text { Last info. } \\
\text { block }\end{array}$ \\
\hline & $-N$ & $-N_{b}$ & & $-N_{b}$ & $N_{e}$ \\
\hline
\end{tabular}

FIGURE 13: Partition of the transmitted data payload for block detection at the receiver.

TABLE 5: Doppler shift estimation in moving-source transmission $(\mathrm{Hz})$.

\begin{tabular}{lccccccc}
\hline \multirow{2}{*}{ Channel } & \multicolumn{7}{c}{ Packet } \\
& 1 & 2 & 3 & 4 & 5 & 6 & 7 \\
\hline 1 & 15.98 & 15.71 & 12.44 & 10.80 & 10.79 & 11.25 & 8.29 \\
2 & 15.98 & 15.71 & 12.44 & 10.80 & 10.79 & 11.25 & 8.38 \\
3 & 15.98 & 15.71 & 12.44 & 10.80 & 10.79 & 11.25 & 8.02 \\
4 & 15.98 & 15.71 & 12.44 & 10.80 & 10.79 & 11.25 & 8.02 \\
5 & 15.98 & 15.71 & 12.44 & 10.80 & 10.79 & 11.25 & 8.46 \\
6 & 16.16 & 15.71 & 12.44 & 10.89 & 10.79 & 11.25 & 8.36 \\
7 & 15.98 & 15.71 & 12.44 & 10.80 & 10.79 & 11.25 & 8.26 \\
8 & 15.98 & 15.71 & 12.44 & 10.80 & 10.79 & 11.25 & 8.29 \\
\hline
\end{tabular}

TABLE 6: Average uncoded BER for Unet06 Experiment.

\begin{tabular}{lcc}
\hline $\begin{array}{l}\text { Number of } \\
\text { combining } \\
\text { channels }\end{array}$ & Moving Source & Fixed Source \\
\hline 1 & $3.027 \mathrm{e}-1$ & $3.135 \mathrm{e}-1$ \\
2 & $2.778 \mathrm{e}-1$ & $2.139 \mathrm{e}-1$ \\
3 & $2.083 \mathrm{e}-2$ & $1.064 \mathrm{e}-2$ \\
4 & $1.017 \mathrm{e}-2$ & $1.740 \mathrm{e}-3$ \\
5 & $3.455 \mathrm{e}-3$ & $1.490 \mathrm{e}-4$ \\
6 & $7.399 \mathrm{e}-4$ & $1.366 \mathrm{e}-4$ \\
7 & $7.222 \mathrm{e}-4$ & $6.621 \mathrm{e}-5$ \\
8 & $6.248 \mathrm{e}-4$ & $3.104 \mathrm{e}-5$ \\
\hline
\end{tabular}

synchronization was achieved and the channel length $L$ was estimated, both with the help of LFMB signal. The equivalent symbol-spaced channel length was estimated as $L=20$ in moving-source transmission. The LFMB signal together with the LFME signal determined the received packet duration, which was used to estimate the average Doppler shift [22]. In Table 5, Doppler shift estimations are listed for seven moving-source packets. For each packet, the estimation was performed independently on each of the eight channels (hydrophones). Obviously, the Doppler shift is nonnegligible compared to the carrier frequency of $17 \mathrm{kHz}$. The visual demonstration of the Doppler effect resorts to the channel scattering function, which was estimated with the $m$-sequence attributing to its sensitivity to Doppler spread. In the left subfigure of Figure 12, the scattering function of the first moving-source packet in Table 5 is demonstrated.
It is obvious that the Doppler spectrum centers around the average Doppler shift. For comparison, the scattering function for a fixed-source packet is also shown in the right subfigure. Since there is no relative transceiver motion, the Doppler spectrum centers around zero in this scenario. The average Doppler shift was compensated in the movingsource packet before detection started.

For the channel length of $L=20$ requiring a minimum training length of 39 with SIMO transmission, a larger training length $N_{p}=200$ was selected. This choice of training length corresponds to a time duration of $50 \mathrm{~ms}$, which is less than the channel coherence time about $200 \mathrm{~ms}$ for a maximum Doppler spread between $2 \sim 3 \mathrm{~Hz}$, as shown in Figure 12 (in moving-source case, the Doppler shift demonstrated in Figure 12(a) will be compensated). The maximum differential phase drift in this case is less than $\pi / 8$ and the approximation for phase drift in (6) is appropriate. Similar to MIMO case, the received packet was partitioned into blocks for processing with a block size of $N_{b}=400$, which is 0.1 seconds of time duration. Only one pilot sequence at the head of the data payload were used to obtain the initial MIMO channel, and the last $N_{p}=$ 200 symbols in the previously-detected block was used to reestimate the channel, leading to the partition structure shown in Figure 13. In the groupwise phase estimation and compensation, the group size of $N_{s}=40$ was selected, and there were $N_{g}=10$ groups in one block.

The scatter plots in Figures 14 and 15 demonstrate the equalized and phase-corrected QPSK symbols for movingsource packet. From Figure 14, the phase rotations in the equalized symbols are even more significant compared to the MIMO results shown in Figures 9 and 10. Regardless of the different experimental environments, the reason lies in the adoption of larger block size of $N_{b}=400$, compared to $N_{b}=$ 200 used in MIMO case, during detection. The selection of larger block size decreases the complexity for channel adaption, while it increases the accumulation of phase rotations in the equalized symbols. From Figure 15, the symbols are well classified after phase correction, which indicates that the adopted phase estimation and compensation algorithm is effective and robust under different channel conditions.

The uncoded BER results for UNet06 experiment are listed in Table 6. In total, seven moving-source packets and six fixed-source packets were processed. The processing procedure for fixed-source packets was similar to that of moving-source packet, except that the channel length was $L=60$ in this case. The listed BERs are averaged ones over 


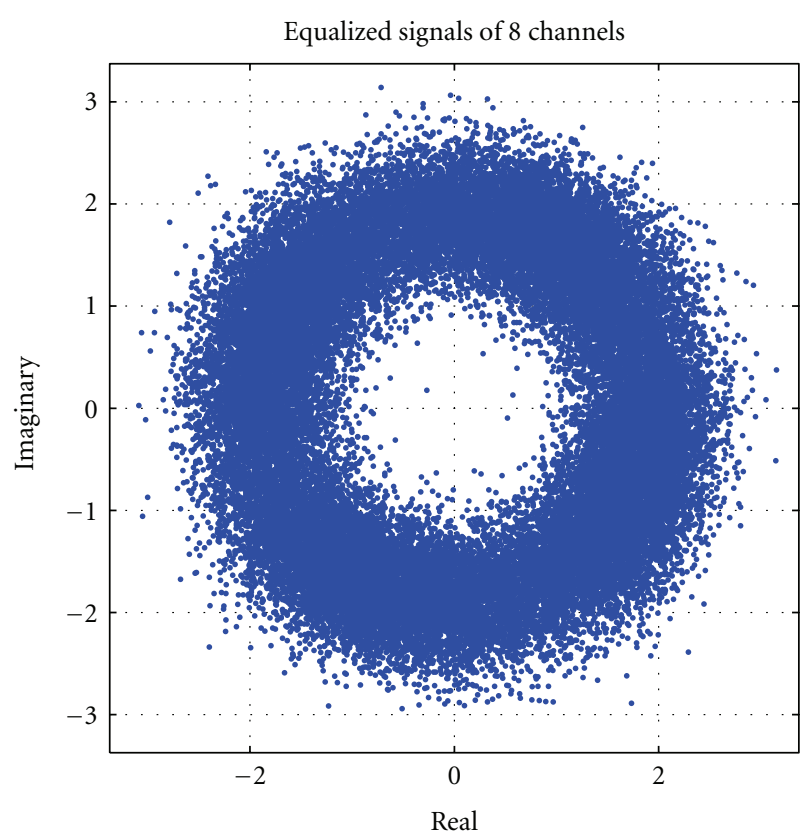

FIGURE 14: Scatter plot of equalized QPSK symbols using eight channels in Unet06 experiment.

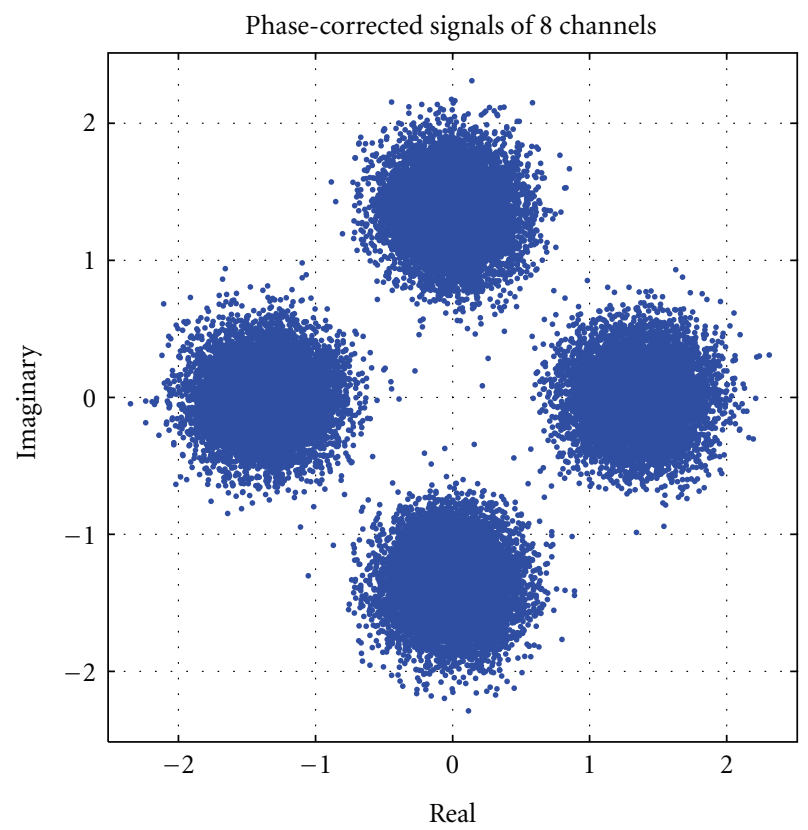

FIGURE 15: Scatter plot of phase-corrected QPSK symbols using eight channels in Unet06 experiment.

all packets. To demonstrate the diversity gain, the results corresponding to different number of combining channels are all shown. Two observations are made. First, the fixedsource transmission has better uncoded BER performance than the moving-source transmission, due to the absence of Doppler shift in the received signal. Second, it is obvious that the uncoded BER decreases when the number of channels used increases due to the increased diversity gain. When all eight channels are used, the best uncoded BER is achieved for both transmissions.

\section{Conclusion}

We have demonstrated a new time-domain MIMO equalization scheme for high data rate single-carrier underwater acoustic communications, where the separate interference cancelation and phase compensation operations have been performed during detection. MIMO linear equalization was operated in an ordered successive interference cancelation fashion to achieve enhanced performance. A novel groupwise phase estimation and compensation algorithm was used to remove the phase distortion in the equalized symbols. MIMO channel was estimated with pilot symbols in training mode and was tracked using previously detected symbols in decision-directed mode, incurring no more than 5\% training overhead, compared to $20 \% \sim 30 \%$ in existing receiver designs. The proposed equalization scheme was tested by extensive experimental data measured off the northwestern coast of Kauai, Hawaii, in September 2005, and at Saint Margaret's Bay, Nova Scotia, Canada, in May 2006. Processing results have shown the effectiveness and robustness of the proposed new equalization scheme under different UWA transmission conditions.

\section{Appendix}

\section{A. Calculation of MIMO LE Matrix}

The MIMO LE matrix given in (12) has implicit forms which are difficult for evaluation. Therefore, it is desirable to provide explicit solutions directly relating to the channel knowledge. To achieve that, we first represent $\mathbf{y}(k-q)$ of (10a) in the following form:

$$
\mathbf{y}(k-q)=\sum_{l=0}^{L-1} \mathbf{H}(l) \mathbf{x}(k-q-l)+\mathbf{v}(k-q)
$$

where $\mathbf{x}(k-q-l)=\left[x_{1}(k-q-l), x_{2}(k-q-l), \ldots, x_{N}(k-\right.$ $q-l)]^{t}, \mathbf{v}(k-q)=\left[v_{1}(k-q), v_{2}(k-q), \ldots, v_{M}(k-q)\right]^{t}$, and

$$
\mathbf{H}(l)=\left[\begin{array}{cccc}
\breve{h}_{1,1}(l) & \breve{h}_{2,1}(l) & \ldots & \breve{h}_{N, 1}(l) \\
\breve{h}_{1,2}(l) & \breve{h}_{2,2}(l) & \ldots & \breve{h}_{N, 2}(l) \\
\vdots & \vdots & \ldots & \vdots \\
\breve{h}_{1, M}(l) & \breve{h}_{2, M}(l) & \cdots & \breve{h}_{N, M}(l)
\end{array}\right]
$$

with $\breve{h}_{n, m}(l)=h_{n, m}(l) e^{j \phi_{n, m}(I)}$ being the effective channel coefficient combining the fading tap with phase drift. Herein, the index $I$ is determined as $I=\left(2 k+K_{1}-K_{2}\right) / 2$ similar to that in (5), and the approximation leading to (6) is also used to validate (A.1). Then, we are able to express $\mathbf{y} \in$ $\mathrm{C}^{M\left(K_{1}+K_{2}+1\right) \times 1}$ in $(9)$ as

$$
\mathbf{y}=\mathbf{H x}+\mathbf{v},
$$

where $\mathbf{H} \in \mathcal{C}^{M\left(K_{1}+K_{2}+1\right) \times N\left(K_{1}+K_{2}+L\right)}$ is given in the following: 


$$
\mathbf{H}=\left[\begin{array}{cccccccc}
\mathbf{H}(L-1) & \mathbf{H}(L-2) & \ldots & \mathbf{H}(0) & \mathbf{0} & \mathbf{0} & \ldots & \mathbf{0} \\
\mathbf{0} & \mathbf{H}(L-1) & \mathbf{H}(L-2) & \ldots & \mathbf{H}(0) & \mathbf{0} & \ldots & \mathbf{0} \\
\vdots & \vdots & \ddots & \ddots & \ddots & \ddots & \vdots & \vdots \\
\mathbf{0} & \mathbf{0} & \ldots & \mathbf{0} & \mathbf{H}(L-1) & \mathbf{H}(L-2) & \cdots & \mathbf{H}(0)
\end{array}\right] \text {, }
$$

and $\mathbf{x}=\left[\mathbf{x}^{t}\left(k-K_{2}-L+1\right), \mathbf{x}^{t}\left(k-K_{2}-L+\right.\right.$ $\left.2), \ldots, \mathbf{x}^{t}\left(k+K_{1}\right)\right]^{t} \in \mathrm{C}^{N\left(K_{1}+K_{2}+L\right) \times 1}$ is a vector containing transmission symbols of all $N$ transducers during the period $\left[k-K_{2}-L+1, k+K_{1}\right]$, and the noise vector is defined as $\mathbf{v}=\left[\mathbf{v}^{t}\left(k-K_{2}\right), \mathbf{v}^{t}\left(k-K_{2}+1\right), \ldots, \mathbf{v}^{t}\left(k+K_{1}\right)\right]^{t} \in$ $\mathrm{C}^{M\left(K_{1}+K_{2}+1\right) \times 1}$. Now, by substituting $\mathbf{y}$ in (12) with (A.3), we obtain

$$
\mathbf{C}_{\text {MMSE }}=\mathbb{E}\left[\mathbf{x}(k) \mathbf{x}^{h}\right] \mathbf{H}^{h}\left\{\mathbf{H} \mathbb{E}\left[\mathbf{x} \mathbf{x}^{h}\right] \mathbf{H}^{h}+\mathbb{E}\left[\mathbf{v} \mathbf{v}^{h}\right]\right\}^{-1} .
$$

In (A.5), we still need to evaluate $\mathbb{E}\left[\mathbf{x} \mathbf{x}^{h}\right], \mathbb{E}\left[\mathbf{x}(k) \mathbf{x}^{h}\right]$. Herein, the assumption that the transmitted symbols are uncorrelated both spatially and temporally is used. In this case, we have $\mathbb{E}\left[x_{i}(k) x_{j}(l)\right]=\sigma_{x}^{2} \delta(i-j) \delta(k-l)$ with $\sigma_{x}^{2}$ being the power of transmission symbol $x_{n}(k)$, and

$$
\mathbf{R}_{x} \triangleq \mathbb{E}\left[\mathbf{x} \mathbf{x}^{h}\right]=\sigma_{x}^{2} \mathbf{I}_{N\left(K_{1}+K_{2}+L\right)} \in \mathfrak{C}^{N\left(K_{1}+K_{2}+L\right) \times N\left(K_{1}+K_{2}+L\right)},
$$

$$
\begin{aligned}
\mathbf{R}^{h} & \triangleq \frac{1}{\sigma_{x}^{2}} \mathbb{E}\left[\mathbf{x}(k) \mathbf{x}^{h}\right] \\
& =\left[\begin{array}{lll}
\mathbf{0}_{N \times\left(K_{2}+L-1\right) N} & \mathbf{I}_{N} & \mathbf{0}_{N \times K_{1} N}
\end{array}\right] \in \mathcal{C}^{N \times N\left(K_{1}+K_{2}+L\right)} .
\end{aligned}
$$

Defining the SNR $\beta$ as

$$
\beta=\frac{\sigma_{x}^{2}}{\sigma_{v}^{2}}
$$

and combining (A.6a)-(A.6b), we finally express the LE matrix in (A.5) as follows:

$$
\begin{aligned}
\mathbf{C}_{\text {MMSE }} & =\mathbf{R}^{h} \mathbf{H}^{h}\left(\mathbf{H} \mathbf{H}^{h}+\frac{1}{\beta} \mathbf{I}_{M\left(K_{1}+K_{2}+1\right)}\right)^{-1} \\
& =\mathbf{R}^{h}\left(\mathbf{H}^{h} \mathbf{H}+\frac{1}{\beta} \mathbf{I}_{N\left(K_{1}+K_{2}+L\right)}\right)^{-1} \mathbf{H}^{h} .
\end{aligned}
$$

\section{Acknowledgments}

This work was supported in part by the Office of Naval Research under Grants N00014-07-1-0219 and N00014-101-0174 and the National Science Foundation under Grants ECCS-0846486 and CCF-0915846. The work of T. C. Yang and W.-B. Yang was supported by the Office of Naval Research. The authors are grateful to Professor Tolga Duman and Dr. Subhadeep Roy for providing the transmitted data of MakaiEx05.

\section{References}

[1] D. B. Kilfoyle and A. B. Baggeroer, "State of the art in underwater acoustic telemetry," IEEE Journal of Oceanic Engineering, vol. 25, no. 1, pp. 4-27, 2000.

[2] D. J. Garrood, "Application of the MFSK acoustical communication system," in Proceedings of the MTS/IEEE Oceans Conference, pp. 67-71, Boston, Mass, USA, 1981.

[3] M. Stojanovic, J. Catipovic, and J. G. Proakis, "Adaptive multichannel combining and equalization for underwater acoustic communications," Journal of the Acoustical Society of America, vol. 94, no. 3, part 1, pp. 1621-1631, 1993.

[4] D. Rouseff, D. R. Jackson, W. L. J. Fox, C. D. Jones, J. A. Ritcey, and D. R. Dowling, "Underwater acoustic communication by passive-phase conjugation: theory and experimental results," IEEE Journal of Oceanic Engineering, vol. 26, no. 4, pp. 821$831,2001$.

[5] G. F. Edelmann, T. Akal, W. S. Hodgkiss, S. Kim, W. A. Kuperman, and H. C. Song, "An initial demonstration of underwater acoustic communication using time reversal," IEEE Journal of Oceanic Engineering, vol. 27, no. 3, pp. 602$609,2002$.

[6] T. C. Yang, "Differences between passive-phase conjugation and decision-feedback equalizer for underwater acoustic communications," IEEE Journal of Oceanic Engineering, vol. 29, no. 2, pp. 472-487, 2004.

[7] M. Stojanovic, J. A. Catipovic, and J. G. Proakis, "Phasecoherent digital communications for underwater acoustic channels," IEEE Journal of Oceanic Engineering, vol. 19, no. 1, pp. 100-111, 1994.

[8] S. Roy, T. Duman, L. Ghazikhanian, V. McDonald, J. Proakis, and J. Zeidler, "Enhanced underwater acoustic communication performance using space-time coding and processing," in Proceedings of the MTS/IEEE Oceans Conference, pp. 26-33, November 2004.

[9] J. W. Choi, R. J. Drost, A. C. Singer, and J. Preisig, "Iterative multi-channel equalization and decoding for high frequency underwater acoustic communications," in Proceedings of the 5th IEEE Sensor Array and Multichannel Signal Processing Workshop, pp. 127-130, July 2008.

[10] T. C. Yang, "Correlation-based decision-feedback equalizer for underwater acoustic communications," IEEE Journal of Oceanic Engineering, vol. 30, no. 4, pp. 865-880, 2005.

[11] J. Tao, Y. R. Zheng, C. Xiao, T. C. Yang, and W.-B. Yang, "Channel estimation, equalization and phase correction for single carrier underwater acoustic communications," in Proceedings of the MTS/IEEE Oceans Conference, pp. 1-6, Kobe, Japan, April 2008.

[12] Y. R. Zheng, C. Xiao, T. C. Yang, and W.-B. Yang, "Frequencydomain channel estimation and equalization for shallowwater acoustic communications," Physical Communication, vol. 3, no. 1, pp. 48-63, 2010. 
[13] M. Stojanovic, "Low complexity OFDM detector for underwater acoustic channels," in Proceedings of the MTS/IEEE Oceans Conference, pp. 1-6, September 2006.

[14] B. Li, S. Zhou, M. Stojanovic, F. L. Freitag, and P. Willett, "Multicarrier communication over underwater acoustic channels with nonuniform Doppler shifts," IEEE Journal of Oceanic Engineering, vol. 33, no. 2, pp. 198-209, 2008.

[15] D. B. Kilfoyle, J. C. Preisig, and A. B. Baggeroer, "Spatial modulation experiments in the underwater acoustic channel," IEEE Journal of Oceanic Engineering, vol. 30, no. 2, pp. 406$415,2005$.

[16] H. C. Song, P. Roux, W. S. Hodgkiss, W. A. Kuperman, T. Akal, and M. Stevenson, "Multiple-input-multiple-output coherent time reversal communications in a shallow-water acoustic channel," IEEE Journal of Oceanic Engineering, vol. 31, no. 1, pp. 170-178, 2006.

[17] S. Roy, T. M. Duman, V. McDonald, and J. G. Proakis, "High-rate communication for underwater acoustic channels using multiple transmitters and space-time coding: receiver structures and experimental results," IEEE Journal of Oceanic Engineering, vol. 32, no. 3, pp. 663-688, 2007.

[18] M. L. Nordenvaad and T. Öberg, "Iterative reception for acoustic underwater MIMO Communications," in Proceedings of the MTS/IEEE Oceans Conference, pp. 1-6, September 2006.

[19] J. Zhang, Y. R. Zheng, and C. Xiao, "Frequency-domain equalization for single carrier MIMO underwater acoustic communications," in Proceedings of the MTS/IEEE Oceans Conference, September 2008.

[20] B. Li, J. Huang, S. Zhou et al., "MIMO-OFDM for highrate underwater acoustic communications," IEEE Journal of Oceanic Engineering, vol. 34, no. 4, pp. 634-644, 2009.

[21] C. Xiao and Y. R. Zheng, "Channel equalization and symbol detection for single carrier broadband MIMO systems with multiple carrier frequency offsets," in Proceedings of the IEEE International Conference on Communications (ICC '08), pp. 4316-4320, Beijing, China, May 2008.

[22] B. S. Sharif, J. Neasham, O. R. Hinton, and A. E. Adams, "Computationally efficient Doppler compensation system for underwater acoustic communications," IEEE Journal of Oceanic Engineering, vol. 25, no. 1, pp. 52-61, 2000.

[23] T. H. Eggen, A. B. Baggeroer, and J. C. Preisig, "Communication over Doppler spread channels_-part I: channel and receiver presentation," IEEE Journal of Oceanic Engineering, vol. 25 , no. 1 , pp. $62-71,2000$.

[24] G. J. Foschini, "Layered space-time architecture for wireless communication in a fading environment when using multielement antennas," Bell Labs Technical Journal, vol. 1, no. 2, pp. 41-59, 1996.

[25] A. Lozano and C. Papadias, "Layered space-time receivers for frequency-selective wireless channels," IEEE Transactions on Communications, vol. 50, no. 1, pp. 65-73, 2002.

[26] P. W. Wolnainsky, G. J. Foschini, G. D. Golden, and R. A. Valenzuela, "V-BLAST: an architecture for achievingvery high data rates over the rich-scattering wireless channel," in Proceedings of the International Symposium on Signals, Systems, and Electronics (ISSSE '98), Pisa, Italy, September 1998.

[27] V. Tarokh, N. Seshadri, and A. R. Calderbank, "Space-time codes for high data rate wireless communication: performance criterion and code construction," IEEE Transactions on Information Theory, vol. 44, no. 2, pp. 744-765, 1998.

[28] M. V. Clark, "Adaptive frequency-domain equalization and diversity combining for broadband wireless communications," IEEE Journal on Selected Areas in Communications, vol. 16, no. 8, pp. 1385-1395, 1998. 